\title{
Implications from Late-time X-Ray Detections of Optically Selected Tidal Disruption Events: State Changes, Unification, and Detection Rates
}

\author{
P. G. Jonker ${ }^{1,2}$ (1) , N. C. Stone ${ }^{3,4,5}$, A. Generozov ${ }^{5,6}$, S. van Velzen ${ }^{7,8}$ (10), and B. Metzger ${ }^{5}$ (1) \\ ${ }^{1}$ SRON, Netherlands Institute for Space Research, Sorbonnelaan 2, 3584 CA, Utrecht, The Netherlands; p.jonker@sron.nl \\ 2 Department of Astrophysics/IMAPP, Radboud University, P.O. Box 9010, 6500 GL, Nijmegen, The Netherlands \\ Department of Astronomy, University of Maryland, Stadium Drive, College Park, MD 20742-2421, USA \\ ${ }^{4}$ Racah Institute of Physics, The Hebrew University, Jerusalem, 91904, Israel \\ ${ }^{5}$ Department of Physics, Department of Astronomy, and Columbia Astrophysics Laboratory, Columbia University, New York, NY 10027, USA \\ 6 JILA, University of Colorado and National Institute of Standards and Technology, 440 UCB, Boulder, CO 80309-0440, USA \\ ${ }^{7}$ Center for Cosmology and Particle Physics, New York University, New York, NY 10003, USA \\ ${ }^{8}$ Joint Space-Science Institute, University of Maryland, College Park, MD 20742, USA \\ Received 2019 June 28; revised 2019 December 13; accepted 2019 December 23; published 2020 February 4
}

\begin{abstract}
We present Chandra X-ray observations of four optically selected tidal disruption events (TDEs) obtained 4-9 yr after discovery. Three sources were detected with luminosities between $9 \times 10^{40}$ and $3 \times 10^{42} \mathrm{erg} \mathrm{s}^{-1}$. The spectrum of PTF09axc is consistent with a power law of index $2.5 \pm 0.1$, whereas the spectrum of PTF09ge is very soft. The power-law spectrum of PTF09axc and prior literature findings provide evidence that TDEs transition from an early-time soft state to a late-time hard state many years after disruption. We propose that the time to peak luminosity for optical and X-ray emission may differ substantially in TDEs, with X-rays being produced or becoming observable later. This delay helps explain the differences in observed properties such as $L_{\mathrm{opt}} / L_{\mathrm{X}}$ of optically and X-ray-selected TDEs. We update TDE rate predictions for the eROSITA instrument: it ranges from 3 to $990 \mathrm{yr}^{-1}$, depending sensitively on the distribution of black hole spins and the time delay between disruption and peak X-ray brightness. We further predict an asymmetry in the number of retrograde and prograde disks in samples of optically and X-ray-selected TDEs. The details of the observational biases can contribute to observed differences between optically and X-ray-selected TDEs (with optically selected TDEs being fainter in X-rays for retrograde TDE disks).
\end{abstract}

Unified Astronomy Thesaurus concepts: Astrophysical black holes (98); Tidal disruption (1696); Active galactic nuclei (16); High energy astrophysics (739); X-ray transient sources (1852)

\section{Introduction}

Stellar tidal disruption is an unavoidable outcome of collisional orbital dynamics in dense stellar systems (Frank \& Rees 1976). The stochastic two-body relaxation of orbital parameters leads stars on a random walk through angular momentum space, eventually delivering them to pericenters close to the supermassive black hole (SMBH). Once a star's orbital pericenter falls within the tidal, or Roche, radius of the $\mathrm{SMBH}$, the star will be destroyed upon pericenter passage (Hills 1975; Rees 1988). The resulting tidal disruption events (TDEs) were theoretical curiosities for many years but have been discovered in increasing numbers over the last two decades. There are now dozens of known TDEs discovered as transient nuclear flares, which have been identified primarily through quasi-thermal emission in soft X-ray (e.g., Bade et al. 1996; Greiner et al. 2000; Komossa et al. 2004; Donato et al. 2014), UV (Gezari et al. 2006, 2009), and optical (van Velzen et al. 2011a, 2019a; Gezari et al. 2012; Arcavi et al. 2014; Chornock et al. 2014; Holoien et al. 2014, 2016a, 2016b) wavelengths. A minority of TDEs have been observed to launch relativistic jets detectable (via nonthermal hard X-ray and soft $\gamma$-ray emission) to cosmological distances (e.g., Bloom et al. 2011; Levan et al. 2011). However, late-time radio follow-up of thermally selected TDEs usually returns upper limits (Bower et al. 2013; van Velzen et al. 2013), suggesting that only a minority of TDEs are accompanied by very high luminosity jets (Generozov et al. 2017).
Astrophysical interest in TDEs is manifold. These flares hold great scientific potential as probes of SMBH demographics, as the mass fallback rate onto the black hole encodes the mass (Rees 1988; Lodato et al. 2009; Guillochon \& RamirezRuiz 2013) of the SMBH. The SMBH spin may be more subtly imprinted into TDE observables (Stone \& Loeb 2012; Guillochon \& Ramirez-Ruiz 2015; Hayasaki et al. 2016). In the subset of TDEs that launch relativistic jets, radio synchrotron emission produced in the jet forward shock can place tight constraints on circumnuclear gas in distant galactic nuclei (Giannios \& Metzger 2011; Berger et al. 2012). More speculatively, these jets could be responsible for the observed flux of ultrahigh-energy cosmic rays (Farrar \& Gruzinov 2009; Farrar \& Piran 2014). Exotic TDEs may serve as signposts of unusual SMBH dynamics: truncated light curves are expected in the vicinity of close SMBH binaries (Liu et al. 2009), and off-nuclear TDEs may indicate SMBHs recoiling after anisotropic gravitational wave emission (Stone \& Loeb 2011; Jonker et al. 2012). Finally, TDEs may also serve as natural accretion physics laboratories, as the mass fallback feeding the disk declines from super-Eddington levels to a few percent of Eddington over a period of months to years (Shen \& Matzner 2014). As TDE accretion rates decline from super-Eddington, to modestly sub-Eddington, to very subEddington levels, their accretion disks might exhibit state changes analogous to those of stellar mass black holes in X-ray binaries (XRBs; Fender et al. 2004; Komossa et al. 2004).

Early models for TDE light curves and spectra assumed that the highly eccentric debris streams from stellar disruption 
would quickly circularize into a compact accretion disk (Rees 1988; Cannizzo et al. 1990; Ulmer 1999) that might resemble a scaled-up XRB disk or the innermost regions of an active galactic nucleus (AGN). A circularized TDE disk would differ from both of these analogs in its radial extent: typically, the tidal radius $R_{\mathrm{t}} \lesssim 100 R_{\mathrm{g}}$, where $R_{\mathrm{g}}$ is the $\mathrm{SMBH}$ gravitational radius, a scale much smaller than the typical XRB or AGN disk.

This simple expectation has, however, been strongly challenged. Recent analytic and numerical theory has found that circularization may be very slow if the debris pericenter $R_{\mathrm{p}} \gg 10 R_{\mathrm{g}}$ (Dai et al. 2015; Piran et al. 2015; Shiokawa et al. 2015) and/or there is strong misalignment between the SMBH spin vector and the debris angular momentum vector (Guillochon \& Ramirez-Ruiz 2015; Hayasaki et al. 2016). In tandem, early-time observations have found four properties characteristic of optical/UV-selected TDEs (van Velzen et al. 2011a; Arcavi et al. 2014; Hung et al. 2018): (i) low blackbody temperatures $\left(T_{\mathrm{BB}} \approx 2 \times 10^{4} \mathrm{~K}\right)$ with blackbody radii $R_{\mathrm{BB}} \sim$ $10^{2-3} R_{\mathrm{g}}$, (ii) little cooling $\left(d \ln \left(T_{\mathrm{BB}}\right) / d t<0.01 \mathrm{day}^{-1}\right)$ over an $\sim 100$ day baseline, (iii) a steep power-law decay in observed flux $F(t)$ often consistent with $F \propto t^{-5 / 3}$, and (iv) very high optical/ UV luminosities, with $L_{\mathrm{BB}} \sim 10^{43.5-44.5} \mathrm{erg} \mathrm{s}^{-1}$ near peak.

All of these properties are inconsistent with the simplest TDE emission model, which assumes emission from radii $\lesssim R_{\mathrm{t}} \sim 10 R_{\mathrm{g}}$ (Ulmer 1999). In this scenario, the optical/UV emission is far down the Raleigh-Jeans tail of the disk spectral energy distribution (SED) and therefore decays slowly in time, $L_{\mathrm{RJ}} \propto T_{\mathrm{BB}} \propto t^{-5 / 12}$ (Lodato \& Rossi 2011). The predicted level of optical/UV luminosity is $L_{\mathrm{opt}} \sim 10^{41} \mathrm{erg} \mathrm{\textrm {s } ^ { - 1 }}$, far lower than observed. These discrepancies have motivated multiple theoretical alternatives for the observed optical/UV emission: photon-driven (Strubbe \& Quataert 2009) or line-driven (Miller 2015) outflows, emission powered by shocks at debris stream self-intersections (Piran et al. 2015), or thermal reprocessing of accretion power by a layer of gas at large radii (Loeb \& Ulmer 1997; Guillochon et al. 2014).

Conversely, soft X-ray observations of TDEs are more qualitatively consistent with the simple picture of a compact accretion disk. Most X-ray detections of TDEs find very soft spectra, consistent with the Wien tail of (multicolor) blackbodies at temperatures $T \lesssim 0.1 \mathrm{keV}$ (Auchettl et al. 2017), like a scaledup version of a high-soft state XRB. However, these X-ray spectra are almost always taken in the first 1 or $2 \mathrm{yr}$ of the flare, when accretion rates are expected to be, at the very least, at a large fraction of the Eddington limit. Notably, many optically selected TDEs go undetected in X-rays (Gezari et al. 2012), and, vice versa, X-ray-selected TDEs often lack optical variability. For instance, the TDE XMMSL1 J074008.2-853927 reported by Saxton et al. (2017) does not show a large enhancement in the optical. Some even show no evidence for enhanced optical emission. For instance, the TDE SDSS J120136.02+300305.5 discovered by Saxton et al. (2012) had an X-ray luminosity of $3 \times 10^{44} \mathrm{erg} \mathrm{s}^{-1}$ at discovery, while the optical spectrum obtained 12 days after the X-ray discovery shows no spectroscopic features (such as broad emission lines) that are usually associated with TDEs. A recent X-ray-discovered source, XMMSL2 J144605.0 +685735 (Saxton et al. 2019), also shows little or no optical emission above the contribution of the nuclear region of the host galaxy.

So far, we have discussed the state of the art in early-time TDE observations, by which we mean observations taken within $2 \mathrm{yr}$ of the peak of the flare. The behavior of TDE disks at late times is relatively underexplored. We note two differences between the early- and late-time phases.

1. The large theoretical uncertainties associated with circularization and disk formation will be less important long after the peak of the mass return rate. A quasicircular disk is a more reasonable approximation at late times, even if initial circularization was inefficient due to weak apsidal precession (Shiokawa et al. 2015) or misaligned SMBH spin (Guillochon \& Ramirez-Ruiz 2015; Hayasaki et al. 2016).

2. The monotonically declining debris fallback rate suggests that at sufficiently late times, TDE disks may pass through the range of sub-Eddington accretion rates that produce a state change in XRB disks (e.g., Giannios \& Metzger 2011; van Velzen et al. 2011b; Tchekhovskoy et al. 2014). This analogy suggests that once TDE accretion rates decline below a few percent of Eddington, X-ray emission may exhibit features of the XRB low/ hard state, such as a primarily nonthermal, hard powerlaw spectrum. Such "SMBH state changes" have not yet been seen in TDEs, although there is one suggestive example: X-ray observations of the TDE in IC 3599 show a transition from a soft to a harder spectrum at late times (Komossa \& Bade 1999).

The search for late-time TDE X-ray emission is further motivated by the recent Hubble Space Telescope discovery of late-time far-UV (FUV) emission in six optically selected TDEs (van Velzen et al. 2019b). In all six cases, the late-time FUV luminosities were well above the levels predicted from extrapolating a naive $\propto t^{-5 / 3}$ power law. The observed slower rate of decline hints at a transition from fallback-dominated to disk-dominated accretion rates (Cannizzo et al. 1990), and the small fitted blackbody radii $\left(R_{\mathrm{BB}} \sim 2-5 R_{\mathrm{t}}\right)$ indicate that if optically thick reprocessing layers once existed, they have since dissipated. It is therefore reasonable to expect that many optically selected TDEs should, at late times, be emitting relatively unobscured X-rays from their inner disks.

In this paper, we present and analyze Chandra observations of four optically selected TDEs taken at late times, long after the peak of the optical flare has passed. We have observed PTF09axc and PTF09ge $8 \mathrm{yr}$ after their discovery, PTF09djl $9 \mathrm{yr}$ after its discovery, and ASASSN-14ae $5 \mathrm{yr}$ after its discovery. In Section 2, we present our observations and results, and in Section 3, we discuss the implications of both detections and nondetections for broader questions in TDE and accretion physics. We adopt $\Omega_{m}=0.3, \Omega_{\Lambda}=0.7$, and $H_{0}=70 \mathrm{~km} \mathrm{~s}^{-1} \mathrm{Mpc}^{-1}$ to convert the redshift of each source to a luminosity distance.

\section{Observations, Analysis, and Results}

We obtained 69.19, 34.15, 9.6, and $19.08 \mathrm{ks}$ long on-source Chandra exposures of PTF09axc, PTF09ge, ASASSN-14ae, and PTF09djl, respectively. The first two sources were observed under Chandra Guest Observer program 18700591 and the latter two under 20700515. The observation of PTF09axc was split into two parts of 53.66 and $15.53 \mathrm{ks}$ in length. The observation identification (ID) numbers for the data presented here are 19532 (53.66 ks) and 20879 (15.53 ks) for PTF09axc, 19531 for PTF09ge, 21503 for ASASSN-14ae, and 21504 for PTF09djl, with observing dates and start times 
Table 1

A Log of the Chandra Late-time X-Ray Observations of Four Optically Selected TDEs

\begin{tabular}{lcccc}
\hline \hline Source & $\begin{array}{c}\text { Observing Date } \\
\text { MJD (UTC) }\end{array}$ & Observation ID & $\begin{array}{c}\text { Duration } \\
(\mathrm{ks})\end{array}$ & $\begin{array}{c}\text { Delay } \\
(\Delta t ; \mathrm{yr})\end{array}$ \\
\hline PTF09axc & $58,095.966$ & 19532 & 53.66 & 8.5 \\
PTF09axc & $58,093.759$ & 20879 & 15.53 & 8.5 \\
PTF09ge & $58,024.847$ & 19531 & 34.15 & 8.4 \\
ASASSN-14ae & $58,439.909$ & 21503 & 9.6 & 4.8 \\
PTF09djl & $58,489.547$ & 21504 & 19.08 & 9.5 \\
\hline
\end{tabular}

Note. The time since the discovery of the optical transient is denoted with $\Delta t$ (delay).

(UTC) of 2017 December 8 at 23:11:32, 2017 December 6 at 18:12:18, 2017 September 28 at 20:19:15, 2018 November 17 at 21:48:37, and 2019 January 6 at 13:08:18, respectively. A log of the observations can be found in Table 1 .

In all cases, the source position as derived in the initial optical outburst was covered by the S3 CCD of the ACIS-S detector array (Garmire 1997). For the observations of PTF09axc and PTF09ge, three CCDs were operational (besides the S3 CCD, S4 and S2 were operational), and the full CCDs were read out, providing a nominal exposure time per frame of $3.1 \mathrm{~s}$. For the observations of ASASSN-14ae and PTF09djl, we chose to use only the S3 CCD. It was operated in subarray mode, where only a quarter of the CCD is read out. This yields an exposure time of $0.8 \mathrm{~s}$ per CCD frame.

We reprocessed and analyzed the data using the CIAO 4.10 software developed by the Chandra X-ray Center and employing CALDB version 4.8.1. To allow for a thorough rejection of events unrelated to the source, such as cosmic-ray hits, the data telemetry mode was set to very faint. Using the CIAO tool wavdetect, we have detected an X-ray source in an image constructed from the $0.3-7 \mathrm{keV}$ data. The position of the X-ray source is consistent with the optical position of the TDE in all three cases where we detected a source close to the expected position (see Table 2). No X-ray source was detected at the location of the optical outburst source in the case of PTF09dj1.

For the detected sources, we calculate the $95 \%$ confidence uncertainty on the Chandra X-ray position using Equation (12) in Kim et al. (2007), which contains the off-axis angle and the detected number of source counts. All of our sources have been detected on-axis, and the number of wavdetect-detected counts is given in Table 2. This internal positional uncertainty has to be supplemented with the external uncertainty, which includes the uncertainty in the satellite aspect solution and the knowledge of the geometry and alignment of the spacecraft and focal plane. Evans et al. (2010) found this external correction to be 0 " 39, which was subsequently found to be underestimated by 0 ". 16 by Roth \& Budavári (2011). The total external $95 \%$ confidence uncertainty of 0 ". 55 needs to be added in quadrature to the internal positional uncertainties given in Table 2 .

We use the CIAO tool specextract to extract a source spectrum for each of the three detected sources separately using the best known optical coordinates for the sources (see Table 2 for references). We created source and background regions centered on the optical position of the sources. The circular source regions have a radius of $2^{\prime \prime}$. The background regions for PTF09axc and PTF09ge are annular with inner and outer radii of $10^{\prime \prime}$ and $30^{\prime \prime}$, respectively. For ASASSN-14ae, the background is drawn from a source-free, circular region on the same CCD (because of the smaller sky area covered due to the employment of a subarray in the readout). This circular region has a radius of $30^{\prime \prime}$. We do not rebin the extracted source spectra, although we require each channel to have at least one $\mathrm{X}$-ray photon. We report the $68 \%$ confidence regions for fitted parameters unless mentioned otherwise.

We fitted the extracted spectra of each source individually using the HEASOFT XSPEC tool version 12.10.1. We excluded photons detected outside the range $0.3-7 \mathrm{keV}$, as this energy interval is the best calibrated and most sensitive range for Chandra. Throughout the spectral fitting, we employ Cash statistics (Cash 1979) unless mentioned otherwise. For each source, we fit the background spectrum separately first. A power law is an adequate first-order description of the background spectrum (see Table 3). When fitting the source spectrum, the background is described using the shape and parameters fixed to those derived from the separate background fit. We scale the normalization of the power-law model (that describes the background) on the basis of the ratio between the size of the source region and that of the background region.

\subsection{PTF09axc}

With a redshift of $z=0.1146\left(d_{L}=532.6 \mathrm{Mpc}\right)$, PTF09axc is associated with the galaxy SDSS J145313.07+221432.2 (Arcavi et al. 2014). Given the relatively high observed count rate of PTF09axc, we investigate whether the source spectrum is affected by pileup by employing the CIAO tool PILEUP_MAP on an image created including all photon energies for both observations of PTF09axc. The count rate per frame in both observations is less than 0.02 , implying a pileup fraction lower than $1 \%$. Therefore, we conclude that pileup is insignificant for our observations of PTF09axc, and by extension, given that the other sources we observed have a lower count rate per frame, those spectra are not affected by pileup either.

In the fit, we take the attenuating effect of Galactic foreground extinction into account. To model this effect, we use the XSPEC PHABS multiplicative model, where we convert the $A_{V}=0.098$ for Galactic foreground extinction obtained through NED (Schlafly \& Finkbeiner 2011) to $N_{\mathrm{H}}=1.8 \times 10^{20} \mathrm{~cm}^{-2}$ using the relation $N_{\mathrm{H}}=1.79 A_{V} \times 10^{21} \mathrm{~cm}^{-2}$ (Predehl \& Schmitt 1995). The value of $N_{\mathrm{H}}$ is kept fixed during the fit. We employ the XSPEC fit function PEGPWR + PHABS $\times$ PEGPWR. Here and below, we note that in all cases, the normalization of the PEGPWR function is equal to the unabsorbed $0.3-7 \mathrm{keV}$ flux.

Fitting the two observations together, the spectrum of PTF09axc is well fit by a power law with index $\Gamma=2.5 \pm$ 0.1 (see Figure 1), with an unabsorbed $0.3-7 \mathrm{keV}$ flux of $(9.5 \pm 0.6) \times 10^{-14} \mathrm{erg} \mathrm{cm}^{-2} \mathrm{~s}^{-1}$ translating to a $0.3-7 \mathrm{keV}$ luminosity $L_{\mathrm{X}}=(3.2 \pm 0.2) \times 10^{42} \mathrm{erg} \mathrm{s}^{-1}$. Here and below, in the calculation of the luminosity uncertainty, we only included the uncertainty in the flux measurement and not that in the distance determination. The observed, absorbed $0.3-7 \mathrm{keV}$ flux is $(8.5 \pm 0.5) \times 10^{-14} \mathrm{erg} \mathrm{cm}^{-2} \mathrm{~s}^{-1}$. The C-statistic of the fit was 226.6 for 223 bins and 221 degrees of freedom. Using the GOODNESS command in XSPEC, we found that $100 \%$ of the realizations yield a lower fit statistic (see Jonker et al. 2005 for an explanation of what GOODNESS values imply). In order to investigate this high value of GOODNESS further, we also fitted the two data sets separately. Whereas the value that we obtain for the power-law index is consistent within the $1 \sigma$ errors, the normalization is only consistent within $2 \sigma$. Given the high 
Table 2

World Coordinate System Information for Our Sample

\begin{tabular}{|c|c|c|c|c|c|c|c|}
\hline Source & Optical Position & Chandra X-ray Position & $\begin{array}{l}\text { 95\% Conf. Internal } \\
\text { Uncert. (arcsec) }\end{array}$ & $\begin{array}{l}\text { Total 95\% Conf. } \\
\text { Uncert. (arcsec) }\end{array}$ & $\begin{array}{l}\text { Offset } \\
(\operatorname{arcsec})\end{array}$ & $\begin{array}{l}\text { Source } \\
\text { Counts }\end{array}$ & $\underset{\mathrm{a}}{\text { References }}$ \\
\hline PTF09axc & $14: 53: 13.06+22: 14: 32.2$ & $14: 53: 13.08+22: 14: 32.169$ & 0.11 & 0.56 & 0.2 & 381 & (1) \\
\hline PTF09axc & $223.30442+22.24228$ & $223.30449+22.24227$ & 0.11 & 0.56 & 0.2 & 381 & (1) \\
\hline PTF09ge & $14: 57: 03.18+49: 36: 40.97$ & $14: 57: 03.18+49: 36: 40.865$ & 0.24 & 0.6 & 0.1 & 43 & (1) \\
\hline PTF09ge & $224.26325+49.61138$ & $224.26326+49.61135$ & 0.24 & 0.6 & 0.1 & 43 & (1) \\
\hline ASASSN-14ae & $11: 08: 40.12+34: 05: 52.23$ & $11: 08: 40.13+34: 05: 53.045$ & 0.56 & 0.78 & 0.8 & 8 & (2) \\
\hline ASASSN-14ae & $167.16717+34.09784$ & $167.16719+34.09807$ & 0.56 & 0.78 & 0.8 & 8 & (2) \\
\hline PTF09djl & $16: 33: 55.94+30: 14: 16.3$ & $\ldots$ & $\ldots$ & $\ldots$ & $\cdots$ & $\cdots$ & (1) \\
\hline PTF09djl & $248.4831+30.23786$ & $\cdots$ & $\ldots$ & $\ldots$ & $\ldots$ & $\ldots$ & (1) \\
\hline
\end{tabular}

Notes.

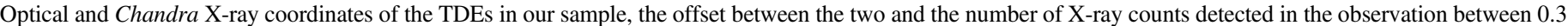

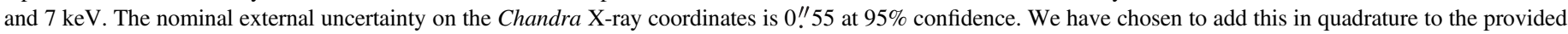

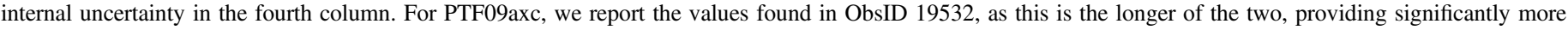
source counts. The coordinates found when using ObsID 20879 are fully consistent with this.

a Reference for the optical coordinates of the sources: (1) Arcavi et al. (2014); (2) Holoien et al. (2014).

number of counts detected, we produced a light curve of the observation with ID 19532 with $1 \mathrm{ks}$ long bins to investigate whether high-amplitude flares are present; none were found, although the source count rate is not constant. Fitting the count rate light curve of $1 \mathrm{ks}$ long bins with a constant yields a reduced $\chi^{2}=2.15$ for 53 degrees of freedom. If spectral variability is associated with this light-curve variability, it could explain the high GOODNESS values of the single observation fit, as well as the spectral fit to the data combined. However, there are not enough X-ray photons detected to investigate this further.

For reference, given the observed number of background events extracted in the background region (1720 for ObsID 19532 and 479 for ObsID 20503), one expects that out of the 447 detected counts at the source position (375 and 72 for the two ObsIDs, respectively), 11 are due to the background (8.5 and 2.4 for the two ObsIDs, respectively).

To check our results, we rebinned the data of ObsID 19532 (the longer of the two observations), requiring that each bin contains at least 30 counts. We subtracted the background and fit the resulting spectrum with a power law attenuated by the foreground Galactic extinction employing $\chi^{2}$ statistics. The result is fully consistent with that obtained by fitting the unbinned data on both data sets.

\subsection{PTF09ge}

With a redshift of $z=0.064\left(d_{L}=287.4 \mathrm{Mpc}\right)$, PTF09ge is associated with the galaxy SDSS J145703.17+493640.9 (Arcavi et al. 2014).

The spectrum of PTF09ge is relatively soft compared to the spectrum of PTF09axc; no photons with energies above $2 \mathrm{keV}$ are detected. We fitted the source spectrum with a redshifted blackbody including a power-law model for the background using Cash statistics. As for PTF09axc, our fit function includes a factor to model the foreground extinction $N_{\mathrm{H}}$. For this, we use a rounded-off value of $1 \times 10^{20} \mathrm{~cm}^{-2}$ given the $A_{V}=0.046$ from NED (Schlafly \& Finkbeiner 2011). The value of $N_{\mathrm{H}}$ is kept fixed during the fit.

Fitting the source and background together, we use a fit function of an absorbed, redshifted blackbody for the source plus a power law for the background (PEGPWR + PHABS $\times$ ZASHIFT $\times$ BBODYRAD in XSPEC). We find a best-fit value for the blackbody temperature of $0.18 \pm 0.02 \mathrm{keV}$. The unabsorbed source flux, subtracting the flux due to the background power law in the $0.3-7 \mathrm{keV}$ range, is $1.9 \times$ $10^{-14} \mathrm{erg} \mathrm{cm}^{-2} \mathrm{~s}^{-1}$, giving a $0.3-7 \mathrm{keV}$ luminosity of $L_{\mathrm{X}}=$ $2 \times 10^{41} \mathrm{erg} \mathrm{s}^{-1}$. The absorbed $0.3-7 \mathrm{keV}$ flux is $\left(1.7_{-0.5}^{+0.3}\right) \times$ $10^{-14} \mathrm{erg} \mathrm{cm}^{-2} \mathrm{~s}^{-1}$. The C-statistic of the fit was 34.5 for 29 bins and 27 degrees of freedom. Using the GOODNESS command in XSPEC, we found that $98 \%$ of the realizations yield a lower fit statistic (when all simulations are drawn from the best-fit model). The bolometric source luminosity of $2.7 \times$ $10^{41} \mathrm{erg} \mathrm{s}^{-1}$ is obtained from the normalization of the blackbody spectral component. Here we assumed that no other emission components are present in other parts of the SED.

As the fit shows some notable residuals, it mostly underpredicts the flux at low energies, so we also try the simple fit function used for PTF09axc (PEGPWR + PHABS $\times$ PEGPWR in XSPEC). For this power-law fit, we find a best-fit value for the power-law index of $3.9 \pm 0.4$ (Figure 1) and an unabsorbed source flux in the $0.3-7 \mathrm{keV}$ range of $3.9_{-0.9}^{+1.2} \times 10^{-14} \mathrm{erg} \mathrm{cm}^{-2} \mathrm{~s}^{-1}$, giving a $0.3-7 \mathrm{keV}$ luminosity of $L_{\mathrm{X}}=3.9_{-1.0}^{+1.1} \times 10^{41} \mathrm{erg} \mathrm{s}^{-1}$. The absorbed $0.3-7 \mathrm{keV}$ flux is $(3.5 \pm 0.9) \times 10^{-14} \mathrm{erg} \mathrm{cm}^{-2} \mathrm{~s}^{-1}$. The C-statistic of the fit was 25.2 for 29 bins and 27 degrees of freedom. Using the GOODNESS command in XSPEC, we found that $58 \%$ of the realizations yield a lower fit statistic (again when all simulations are drawn from the best-fit model).

\subsection{ASASSN-14ae}

With a redshift of $z=0.043671\left(d_{L}=193.3 \mathrm{Mpc}\right)$, ASASSN$14 \mathrm{ae}$ is associated with the galaxy SDSS J110840.11+340552.2 (Holoien et al. 2014). For foreground extinction, $N_{\mathrm{H}}$, we use a rounded-off value of $1 \times 10^{20} \mathrm{~cm}^{-2}$ given the $A_{V}=0.048$ from NED (Schlafly \& Finkbeiner 2011). The value of $N_{\mathrm{H}}$ is kept fixed during the fit.

Eight photons are detected at a position consistent with that of the optical source in outburst. Owing to the relatively short exposure compared to the other observations we report on in this manuscript, on average, only 0.3 background counts would fall in the source extraction region. Given this very low background event rate, the eight-count detection is highly significant; i.e., it occurs due to chance in approximately one out of $8 \times 10^{8}$ cases. For our spectral analysis of these eight photons, we do not correct for this expected background. We fitted for the power-law index and normalization in the fit 
Table 3

X-Ray Spectral Fit Parameters for PTF09axc, PTF09ge, and ASASSN-14ae

\begin{tabular}{lccccc}
\hline \hline Source & $\begin{array}{c}\text { Background } \\
\text { Power-law Index } \Gamma\end{array}$ & $\begin{array}{c}\text { Background } \\
\text { Flux erg cm }{ }^{-2} \mathrm{~s}^{-1}\end{array}$ & $\begin{array}{c}\text { Source Model } \\
\text { Power-law Index } \Gamma\end{array}$ & $\begin{array}{c}\text { Source Flux (Absorbed; 0.3-7 keV) } \\
\text { Flux erg cm }{ }^{-2} \mathrm{~s}^{-1}\end{array}$ & $\begin{array}{c}\text { Luminosity (0.3-7 keV) } \\
\mathrm{erg} \mathrm{s}^{-1}\end{array}$ \\
\hline PTF09axc & $0.7 \pm 0.1$ & $3.2 \times 10^{-16}$ & $2.5 \pm 0.1$ & $(8.5 \pm 0.5) \times 10^{-14}$ & $(3.2 \pm 0.2) \times 10^{42}$ \\
PTF09ge & $0.3 \pm 0.2$ & $3.7 \times 10^{-16}$ & $3.9 \pm 0.4$ & $(3.5 \pm 0.9) \times 10^{-14}$ & $3.9_{-1.0}^{+1.1} \times 10^{41}$ \\
ASASSN-14ae & $\cdots$ & $\cdots$ & $3.2 \pm 1.0$ & $(1.8 \pm 0.8) \times 10^{-14}$ & $(9.5) \times 10^{40}$ \\
PTF09dj1 & $\cdots$ & $\cdots$ & $2.5^{\text {a }}$ & $<3 \times 10^{-15}$ & $<3 \times 10^{41}$ \\
\hline
\end{tabular}

Note. The normalization of the background has been scaled down to match the source photon extraction area (the scaling factor was 200 for PTF09axc and PTF09ge and 900/4 for the circular background region for ASASSN-14ae).

${ }^{\text {a }}$ Parameter fixed to this value. When using a $0.18 \mathrm{keV}$ blackbody to convert the derived upper limit on the number of source photons to flux, the same flux limit as reported for the power-law spectral shape is obtained.

function PHABS $\times$ PEGPWR in XSPEC. The best-fit power-law index is $\Gamma=3.2 \pm 1.0$. The unabsorbed $0.3-7 \mathrm{keV}$ flux is $\left(2_{-1}^{+2}\right) \times 10^{-14} \mathrm{erg} \mathrm{cm}^{-2} \mathrm{~s}^{-1}$, giving a $0.3-7 \mathrm{keV}$ luminosity of $\left(9_{-5}^{+9}\right) \times 10^{40} \mathrm{erg} \mathrm{s}^{-1}$. The absorbed $0.3-7 \mathrm{keV}$ flux is $(1.8 \pm$ $0.8) \times 10^{-14} \mathrm{erg} \mathrm{cm}^{-2} \mathrm{~s}^{-1}$. The C-statistic of the fit was 17.6 for eight bins and six degrees of freedom. Using the GOODNESS command, we found that $96 \%$ of the realizations yield a lower fit statistic (again when all simulations are drawn from the best-fit model). This high GOODNESS shows that the spectral shape is ill constrained given the low number of detected X-ray photons.

\subsection{PTF09djl}

With a redshift of $z=0.184\left(d_{L}=893.2 \mathrm{Mpc}\right)$, PTF09djl is associated with the galaxy SDSS J163355.96+301416.6 (Arcavi et al. 2014). For foreground extinction, $N_{\mathrm{H}}$, we use a rounded-off value of $1 \times 10^{20} \mathrm{~cm}^{-2}$ given the $A_{V}=0.049$ from NED (Schlafly \& Finkbeiner 2011). The value of $N_{\mathrm{H}}$ is kept fixed during the fit.

No X-ray photons with energies between 0.3 and $7 \mathrm{keV}$ have been detected in a circle with a radius of $1^{\prime \prime}$ centered on the optical outburst position of PTF09djl. We estimate the average background photon rate of $0.3-7 \mathrm{keV}$ by extracting the detected counts in a circular region with a radius of $30^{\prime \prime}$ close to the source, where no sources were found when using the WAVDETECT tool with default parameters. In such a region, centered on coordinates R.A. 16:33:52.17, decl. +30:13:44.9, 110 background photons are detected, implying that, on average, 0.12 background counts are expected in a $1^{\prime \prime}$ circular region.

Following Kraft et al. Helene (1984) and (1991) we derive a $95 \%$ confidence upper limit on the number of detected source counts in the $0.3-7 \mathrm{keV}$ band of three. To convert this to a limit on the flux, we divide the upper limit on the detected number of source counts by the on-source time of this observation to obtain an upper limit on the source count rate. Next, we use two models for the spectral shape of the source: a blackbody with a temperature of $180 \mathrm{eV}$, similar to that found for PTF09ge, and a power law with an index of 2.5, as was found for PTF09axc. The attenuating effect of the $N_{\mathrm{H}}$ derived above is marginal and therefore ignored. For both the power-law model and the blackbody model, W3PIMMS ${ }^{9}$ provides a 95\% upper limit to the (absorbed) $0.3-7 \mathrm{keV}$ X-ray flux of $F_{\mathrm{X}} \leqslant 3 \times$ $10^{-15} \mathrm{erg} \mathrm{cm}^{-2} \mathrm{~s}^{-1}$. This yields an upper limit to the source $0.3-7 \mathrm{keV}$ luminosity of $L_{\mathrm{X}} \leqslant 3 \times 10^{41} \mathrm{erg} \mathrm{s}^{-1}$ for both models.

\footnotetext{
9 https://heasarc.gsfc.nasa.gov/cgi-bin/Tools/w3pimms/w3pimms.pl
}

\section{Discussion}

We observed four optically selected TDEs in X-rays using the Chandra satellite. One source, ASASSN-14ae, was observed $4.8 \mathrm{yr}$ after its discovery by Holoien et al. (2014), while the other three sources were observed $\approx 8-10 \mathrm{yr}$ after their discovery in 2009 (note that these three TDEs were reported in the literature $5 \mathrm{yr}$ after their discovery by Arcavi et al. 2014). Three of the four sources were detected; only PTF09djl remains undetected. The X-ray detections of PTF09axc and PTF09ge are especially interesting in conjunction: the X-ray spectrum of PTF09axc is well fit with a power law $(\Gamma=2.5 \pm 0.1)$; conversely, our observations of PTF09ge are well fit by a very soft, $\Gamma=3.9 \pm 0.4$, power law. Finally, for ASASSN-14ae, the number of detected X-ray photons is too low for a meaningful spectral fit. Our Chandra detections are consistent with the 2014 upper limit of $L_{X}<2.3 \times$ $10^{42} \mathrm{erg} \mathrm{s}^{-1}$ for PTF09ge, the 2014 detection of $L_{\mathrm{X}}=7.1_{-3.1}^{+12} \times$ $10^{42} \mathrm{erg} \mathrm{s}^{-1}$ for PTF09axc (Arcavi et al. 2014), and the 2014 upper limit of $L_{\mathrm{X}}<1.3 \times 10^{41} \mathrm{erg} \mathrm{s}^{-1}$ for ASASSN-14ae (Holoien et al. 2014).

Our results indicate that optically selected TDEs may maintain a substantial X-ray luminosity for at least $\sim 5-10 \mathrm{yr}$ post-peak, long after the optical emission has become undetectable. Notably, several optically selected TDEs have stringent early-time X-ray upper limits around or below the luminosities seen in the three sources we detected at late times. For instance, Gezari et al. (2012) provided a nondetection for the optical/UV-selected TDE PS1$10 \mathrm{jh}$, with an upper limit to the $0.2-10 \mathrm{keV}$ X-ray luminosity of $<5.8 \times 10^{41} \mathrm{erg} \mathrm{s}^{-1}$. Blagorodnova et al. (2017) reported a marginal detection of the TDE iPTF16fnl in stacked observations with a $0.3-10 \mathrm{keV}$ luminosity of $2.4_{-1.1}^{+1.9} \times 10^{39} \mathrm{erg} \mathrm{s}^{-1}$, and Hung et al. (2017) did not detect the TDE iPTF16axa down to a $0.3-10 \mathrm{keV}$ luminosity limit of $<3 \times 10^{41} \mathrm{erg} \mathrm{s}^{-1}$. As a caveat, we note that these reported upper limits were provided for the 0.2 / $0.3-10 \mathrm{keV}$ band, whereas in Table 3, we report $0.3-7 \mathrm{keV}$ luminosities. For spectral shapes with a power-law index of 2 (typically assumed for the above cases), these upper limits would be $10 \%-20 \%$ lower when converted to the $0.3-7 \mathrm{keV}$ band.

The late-time detection of X-ray emission in PTF09axc and PTF09ge provides further evidence against the alternative hypothesis that most claimed TDE candidates are, in reality, exotic nuclear supernovae (SNe; Saxton et al. 2018). SNe explosions are not generally bright in X-ray wavelengths, and even among those that are X-ray bright, none are observed to emit above $\sim 10^{39}$ erg s $\mathrm{s}^{-1}$ at times $\gtrsim 10^{4}$ days post-peak (Dwarkadas \& Gruszko 2012). This upper limit is far below even the late-time luminosity detected for PTF09axc and PTF09ge. However, SN2006JD was observed at a luminosity 
of $(2.77 \pm 0.45) \times 10^{41} \mathrm{erg} \mathrm{s}^{-1}$ nearly $4.5 \mathrm{yr}$ after the $\mathrm{SN}$ was first detected (Chandra et al. 2012). This luminosity, as well as the time after the outburst discovery, is consistent with those of ASASSN-14ae. Therefore, in that case, its late-time X-ray luminosity alone is not sufficient evidence for it being a TDE; however, the combined properties (e.g., such as presented in Holoien et al. 2014) and the late-time X-ray luminosity seem incompatible with a SN IIn. Our observations complement late-time FUV detections of six TDE candidates (including PTF09ge) by van Velzen et al. (2019b), which also argue against a "peculiar SNe" interpretation for those sources.

Our results also constrain the hypothesis that PTF09axc may represent extreme optical variability in a low-luminosity AGN. This interpretation was first raised in Arcavi et al. (2014), who observed a weak [O III] emission feature with luminosity $L_{\text {[O III] }}=(2.4 \pm 0.3) \times 10^{39} \mathrm{erg} \mathrm{s}^{-1}$. This feature is not conclusive evidence of an AGN and could also be produced by star formation, but, in conjunction with the 2014 X-ray detection of the host galaxy, it has cast doubt on the TDE status of PTF09axc (see, e.g., Auchettl et al. 2017). Our X-ray luminosity measurement strengthens the case that PTF09axc is indeed a bona fide TDE. Using an empirical relationship between the [O III] and $3-20 \mathrm{keV}$ luminosities in AGNs (Heckman et al. 2005), we can estimate the range of [O III] line luminosities expected if our X-ray detection were of AGN origin (the scatter in this relationship is $\sigma=0.51$ dex, i.e., a factor of $\approx 3.24$ ). Converting our $0.3-7 \mathrm{keV}$ luminosity to the 3-20 keV band using W3PIMMS, PTF09axc has an $L_{\mathrm{X}}(3-20$ $\mathrm{keV})$ of $8 \times 10^{42} \mathrm{erg} \mathrm{s}^{-1}$, and therefore the predicted AGN luminosity for the [O III] line would be $L_{[\mathrm{O} \mathrm{III}]} \approx 5.7 \times$ $10^{40} \mathrm{erg} \mathrm{s}^{-1}$, which is a factor of $\approx 24$ higher than the actual $L_{\text {[O III] }}$ measured by Arcavi et al. (2014). The predicted value of $L_{[\mathrm{O} \text { III }]}$ is inconsistent with the observed value at the $2.7 \sigma$ level, making a conventional AGN origin for the X-ray and [O III] luminosity unlikely.

The detected Chandra luminosities of PTF09ge and ASASSN-14ae can be compared with the late-time FUV luminosities reported for those sources by van Velzen et al. (2019b). The FUV detections of these sources were used to produce disk models and estimates for a range of thermal soft $\mathrm{X}$-ray luminosities; the range of modeled X-ray predictions is particularly sensitive to the dimensionless SMBH spin parameter, $\chi_{\text {. }}$. While our detection of ASASSN-14ae is compatible with the lower end (i.e., retrograde disk and large $|\chi|$.$) of the predicted range \log _{10}\left[L_{X} /\left(\mathrm{erg} \mathrm{s}^{-1}\right)\right]=41.7_{-0.9}^{+1.3}$, our detection of PTF09ge is considerably brighter than the predicted range $\log _{10}\left[L_{\mathrm{X}} /\left(\mathrm{erg} \mathrm{s}^{-1}\right)\right]=37.0_{-2.6}^{+3.6}$ (van Velzen et al. 2019b, where the fiducial predictions correspond to assuming $\chi_{\bullet}=0$, and the lower and upper error bars correspond to assuming $\chi_{\bullet}=-0.9$ and 0.9 , respectively). This discrepancy could be reconciled by invoking even larger values of prograde SMBH spin and/or an SMBH mass somewhat smaller than the fiducial prediction of the $M_{-}-\sigma$ relationship (Wevers et al. 2019b). Unfortunately, PTF09axc was not observed at late times in the FUV.

Interestingly, PTF09djl, which went undetected in the X-ray (with a $0.3-7 \mathrm{keV}$ upper limit of $3 \times 10^{41} \mathrm{erg} \mathrm{s}^{-1}$ ), was detected in the FUV at $3 \times 10^{42} \mathrm{erg} \mathrm{s}^{-1}$, leading to a predicted $\mathrm{X}$-ray luminosity range $\log _{10}\left[L_{\mathrm{X}} /\left(\mathrm{erg} \mathrm{s}^{-1}\right)\right]=41.5_{-1.1}^{+1.6}$. Our nondetection is compatible with this prediction for any range of retrograde SMBH spin values. While there are a number of important caveats associated with the late-time X-ray luminosity predictions from van Velzen et al. (2019b), the strong sensitivity of quasi-thermal $\mathrm{X}$-ray emission to $\chi_{\bullet}$. in latetime TDE disks underlines the value of multiwavelength, latetime observations for constraining SMBH spin. We will return to this subject in Section 3.4.

The late-time X-ray detections of the optically selected TDEs PTF09axc, PTF09ge, and ASASSN-14ae can be compared with late-time detections of X-ray-selected TDEs such as the TDE in IC 3599 reported by Komossa \& Bade (1999). These authors reported that this source underwent a spectral change to a hard spectral state, and at 6-6.5 yr after the X-ray outburst was discovered, the luminosity was $\approx 4 \times 10^{40} \mathrm{erg} \mathrm{s}^{-1}$. Similarly, the TDE in the dwarf galaxy RBS 1032 was found at a luminosity of $10^{41} \mathrm{erg} \mathrm{s}^{-1} 19 \mathrm{yr}$ after discovery (Maksym et al. 2014). Vaughan et al. (2004) presented Chandra X-ray observations of five ROSAT-discovered TDE flares (WPVS 007, IC 3599, RX J1242.6 -1119, RX $1624.9+7554$, and NGC 5905) ranging between $\sim 9$ and $12 \mathrm{yr}$ after their discovery. The late-time luminosities of the sources range from $6 \times 10^{39}$ to $2 \times 10^{41} \mathrm{erg} \mathrm{s}^{-1}$. Out of the five sources, these authors could only provide a spectral fit for IC 3599, which showed that the source power-law index was still rather soft $(\Gamma \approx 3.6)$ even at $\sim 12 \mathrm{yr}$ after discovery. Where the spectral shape of the other four sources derived from broadband colors was likely somewhat harder, the low number of detected counts precluded more detailed spectroscopic fits. Overall, the situation is comparable to the late-time X-ray luminosities and spectral shapes we report for the optically detected TDEs PTF09axc, PTF09ge, and ASASSN-14ae. Apparently, whether or not a TDE is detected in X-rays at early times does not influence the late-time X-ray luminosity.

\subsection{Disk State Changes}

Stellar mass black holes that accrete from companion stars are visible as XRBs. The X-ray emission from these disks exhibits a wide variety of spectral properties, or "states" (e.g., Hasinger \& van der Klis 1989; Fender \& Belloni 2004). ${ }^{10}$ Two of the most commonly observed states, high-soft and low-hard, are characterized by quasi-thermal and power-law spectra, respectively. Soft states often show subdominant power-law $\mathrm{X}$-ray contributions from thermal seed photons upscattered by an electron corona. One of the important variables controlling the accretion state of an XRB disk is the dimensionless mass accretion rate $\dot{m} \equiv \dot{M} / \dot{M}_{\text {Edd }}$, where $\dot{M}$ is the physical accretion rate and $\dot{M}_{\text {Edd }}$ is the Eddington-limited accretion rate. Because $\dot{M}$ in XRB disks can vary greatly on humanly observable timescales, state changes are often observed, typically following a hysteresis pattern (Maccarone \& Coppi 2003). When a source in a high-soft state experiences a persistent decline in $\dot{m}$, it will typically transition to a low-hard state once $\dot{m}$ falls below a threshold value of $\sim 0.03$ (Maccarone \& Coppi 2003). However, some variation in this transition luminosity (as a fractional Eddington luminosity) has been observed: Kalemci et al. (2013) found a soft-to-hard X-ray state change at an Eddington ratio of $\dot{m}=0.0030 \pm 0.0041$, and on the extreme end, Chauhan et al. (2019) found a recent outburst of the candidate black hole XRB MAXI J1535-571 in which the softto-hard spectral state change seems to occur at a fraction $1.2-3.3 \times 10^{-5}$ of the Eddington luminosity (see also

\footnotetext{
${ }^{10}$ Formally, both timing and spectral properties are necessary for the identification of states (Hasinger \& van der Klis 1989). Regrettably, the low number of detected X-ray photons in our late-time TDE observations precludes us from a meaningful X-ray timing study.
} 
Maccarone 2003 for a discussion of variation in Eddington fraction for state changes in XRBs).

There is some evidence that analogous state changes occur in AGN accretion disks around SMBHs (e.g., Maccarone et al. 2003, and references therein). However, as the viscous times in AGN disks are typically much longer than reasonable observational baselines, it is not easy to observe state changes in AGNs. A further difficulty is that in the soft X-rays, AGN spectra are generally dominated by power-law or reflection contributions. This is because the peak of the thermal blackbody disk emission occurs in the extreme UV, where observations are hindered by gas and dust extinction (although a soft spectral component can sometimes be discerned; e.g., Done et al. 2012).

Compared to standard AGNs, TDE disks are probably more favorable laboratories for observing "scaled-up" state changes around SMBHs (Giannios \& Metzger 2011; Tchekhovskoy et al. 2014). The main reason is that the accretion disks expected to form in TDEs are much smaller than AGN disks, implying shorter timescales. If we consider a steady-state Shakura-Sunyaev disk with dimensionless viscosity $\alpha$, constant aspect ratio $H / R$, and outer edge $R_{\mathrm{d}}$, the viscous time scales as $\propto R_{\mathrm{d}}^{3 / 2}$. Late-time TDE disks should be geometrically thin and mostly circularized and have an outer radius $R_{\mathrm{d}} \sim$ $2 R_{\mathrm{p}}=2 R_{\mathrm{t}} / \beta$, where $\beta \sim 1$ is the penetration parameter of the TDE, and the tidal radius is

$$
\begin{aligned}
R_{\mathrm{t}} & =R_{\star}\left(\frac{M_{\bullet}}{M_{\star}}\right)^{1 / 3} \\
& \approx 2 \times 10^{-6} \mathrm{pc}\left(\frac{M \bullet}{10^{6} M_{\odot}}\right)^{1 / 3}\left(\frac{M_{\star}}{M_{\odot}}\right)^{-1 / 3}\left(\frac{R_{\star}}{R_{\odot}}\right) .
\end{aligned}
$$

Here $M_{\star}$ and $R_{\star}$ are the mass and radius of the victim star, and we see that both $R_{\mathrm{t}}$ and $R_{\mathrm{d}}$ are far smaller than the typical radius of an AGN accretion disk, for example, if we take the extent of an AGN broad-line region as a first-order approximation of the size of the AGN accretion disk (e.g., Czerny \& Hryniewicz 2011) using the relation that the 5100 luminosity $\lambda L_{\lambda}$ implies a typical scale $R_{\mathrm{BLR}} \approx 0.026\left(\frac{\lambda L_{\lambda}(5100)}{10^{44}}\right)^{0.7}$ pc (Kaspi et al. 2000), a factor of $10^{4}$ times larger than the typical TDE disk.

Shortly after disruption, the peak mass fallback rate onto the SMBH will generally be super-Eddington, with a peak fallback rate $\dot{M}_{\text {peak }}=\frac{1}{3} M_{\star} / t_{\text {fall }}$, where

$$
t_{\text {fall }} \approx 3.5 \times 10^{6} \mathrm{~s}\left(\frac{M \bullet}{10^{6} M_{\odot}}\right)^{1 / 2}\left(\frac{M_{\star}}{M_{\odot}}\right)^{-1}\left(\frac{R_{\star}}{R_{\odot}}\right)^{3 / 2}
$$

is the fallback time for the most tightly bound debris. In Eddington units, this is (Stone et al. 2013)

$$
\frac{\dot{M}_{\text {peak }}}{\dot{M}_{\text {Edd }}} \approx 130\left(\frac{M \cdot}{10^{6} M_{\odot}}\right)^{-3 / 2}\left(\frac{M_{\star}}{M_{\odot}}\right)^{2}\left(\frac{R_{\star}}{R_{\odot}}\right)^{-3 / 2} .
$$

If circularization is efficient, the disk accretion rate $\dot{M}$ will track the (super-Eddington) mass fallback rate; therefore, the most relevant stellar mass point of comparison might seem to be ultraluminous X-ray sources (ULXs), rather than high-soft XRBs (which are generally sub-Eddington). Contrary to this supposition, early-time soft X-ray detections of TDE candidates generally find quasi-thermal spectra that are analogous to a high-soft state (Komossa \& Greiner 1999; Greiner et al. 2000), particularly in TDEs with good-quality early-time X-ray spectra (e.g., Saxton et al. 2012; Lin et al. 2015; Miller et al. 2015; Holoien et al. 2016a; Gezari et al. 2017; Wevers et al. 2019a), although we note that given the limited passband (typically $0.2-10 \mathrm{keV}$ at best), and especially in cases where the number of detected X-ray photons is below $\sim 10,000$, it is difficult to rule out the soft ULX state. For high signal-to-noise spectra, the main difference found by Gladstone et al. (2009) is that the ULX state is characterized by a high Comptonization optical depth ( $\tau \sim 5-20)$, whereas in the XRB soft state, when fitted with the same model, $\tau$ is always $\leqslant 1$.

However, even in the limiting case of rapid circularization, the super-Eddington phase is expected to last only a fraction of the time TDEs are typically observed. Given the absence of observed state changes from a super-Eddington, ULX-like state to a sub-Eddington, high-soft state, we deem it likely that $\mathrm{X}$-ray-bright TDEs are seen mostly in the equivalent of the XRB soft state. As we will discuss in Section 3.2, the absence of super-Eddington emission may be related to a delay before the sources are detected in X-rays. A soft, quasi-thermal spectrum will no longer be a reasonable expectation (i) at late enough times, once $\dot{m}$ becomes very sub-Eddington, or (ii) if circularization is highly inefficient and $\dot{m} \ll 1$ always. Because $\dot{M} / \dot{M}_{\text {Edd }}$ steadily decreases during the late stages of a TDE flare, we may expect a late-time transition to the $\mathrm{SMBH}$ equivalent of the XRB low-hard state.

Observationally, TDE candidates with soft spectra containing additional hard power-law X-ray spectral components do exist (e.g., Holoien et al. 2016b; Saxton et al. 2017), much like XRB soft states, where a subdominant power-law component also exists. Another example is the X-ray-selected TDE 2XMMi J184725.1-631724 (Lin et al. 2011). It showed an $\mathrm{X}$-ray spectrum that was well fit by a soft thermal component with a temperature of approximately $60 \mathrm{eV}$ plus a (soft) power law with a photon index of around 3-4 contributing around $10 \%-15 \%$ to the total $0.2-10 \mathrm{keV}$ luminosity (at the first detection of the outburst in 2006 September). The temperature of the soft component had risen to around $90 \mathrm{eV} 9$ months later, as measured by XMM-Newton, with a power-law contribution of 5\%-10\%. The X-ray spectrum in the TDE candidate RX J1242-1119 changed from a power law with $\Gamma \approx 5$ (so, a very soft spectrum that could also be fit with a blackbody with a temperature of $0.06 \mathrm{keV}$ ) to $\Gamma \approx 2.5$ at late times (Komossa \& Greiner 1999; Komossa et al. 2004), signifying a potential state change. Finally, the X-ray spectrum of the TDE AT 2018fyk is well fit when a power law is added to the fit function. This power-law component constitutes $\approx 30 \%$ of the unabsorbed flux (Wevers et al. 2019a).

Thus, well-studied TDE X-ray spectra are qualitatively closer to an XRB high-soft state than they are to AGN power laws. The reasons for this are unclear but likely involve the higher blackbody temperature of TDE disks near the innermost stable circular orbit (ISCO), due to (i) the smaller SMBH masses in TDEs relative to most AGNs (compare the SMBH mass distributions in Woo \& Urry 2002; Wevers et al. 2017, 2019b), (ii) the higher early-time Eddington fraction expected for TDEs in comparison to typical AGNs (Kauffmann \& Heckman 2009), and (iii) a bias toward prograde spinning SMBHs for X-ray-selected TDEs (see Section 3.3) enabling a smaller value for the ISCO. Early-time TDE X-ray spectra 
often appear even more thermally dominated than the typical XRB high-soft state, possibly indicating difficulty in forming a Compton scattering corona.

Our interpretation of the spectral properties of PTF09axc and PTF09ge follows straightforwardly from the XRB analogy: PTF09axc has undergone a state change to the SMBH analog of the low-hard state, but this type of change has not yet occurred for PTF09ge, which likely remains in an analog of the high-soft state. This hypothesis is complicated by the Eddington ratios we observe. Using literature estimates for the SMBH masses (Wevers et al. 2019b) and accounting for both the $1 \sigma$ scatter of the underlying $M_{\bullet}-\sigma$ relation and the uncertainty in our X-ray luminosity estimates, we find that PTF09axc was observed at an Eddington fraction of $\dot{m}=$ $5.4_{-3.8}^{+12} \times 10^{-2}$, PTF09ge at $\dot{m}=1.6_{-1.1}^{+3.3} \times 10^{-3}$, and ASASSN14 ae at $\dot{m}=2.8_{-2.4}^{+13} \times 10^{-3}$. The simplest theoretical expectation might be that the TDE disk with the lower Eddington ratio, PTF09ge, should have undergone a state change prior to one with a higher Eddington ratio (PTF09axc). However, we note that in $\mathrm{XRBs}$, the emergence of a coronal power law and the ensuing state change is regulated not only by the accretion rate $\dot{m}$ but also by an additional parameter (see Homan et al. 2001, where the second parameter is interpreted as the fractional size of the Comptonizing region). Furthermore, TDEs differ from standard accretion disks in several ways, and there are other plausible "hidden variables" that may be acting to prevent the emergence of a corona in PTF09ge. For example, the relatively weak magnetic fields of main-sequence stars may mean that TDE disks are born with extremely low magnetizations. ${ }^{11}$ Since coronal electron populations are thought to be accelerated to relativistic energies in magnetic reconnection events (Merloni \& Fabian 2001), standard low-hard state coronas may only emerge in TDE disks born with unusually large magnetizations or ones where external factors like large and retrograde SMBH spin (Parfrey et al. 2015) favor magnetic field generation in situ through dynamo processes.

Overall, the X-ray Eddington ratio of PTF09axc is broadly compatible with the common range of Eddington ratios where soft-to-hard state changes occur in XRBs. The persistently soft spectrum of PTF09ge is more unusual, but as mentioned before, XRB soft states have been observed to persist down to an Eddington ratio of $\sim 10^{-3}$ and, in an extreme case, even down to a few times $10^{-5}$. As an alternative, the different X-ray spectral shape between sources and, in some cases, the changing X-ray spectral slope in one source can also be reproduced by changing parameters involving a different physical model. One possibility includes varying the Comptonization optical depth and/or the disk temperatures of the seed photons of such a Comptonization model.

One testable prediction of our XRB analogy is the predicted radio luminosity using the fundamental plane of black hole activity (Merloni et al. 2003; Falcke et al. 2004). Using the calibration of Merloni et al. (2003), and given the SMBH mass estimate of $\log M_{\bullet}=5.68$ in PTF09axc from Wevers et al. (2017), we derive an expected radio luminosity at $5 \mathrm{GHz}$ of $2 \times 10^{37} \mathrm{erg} \mathrm{s}^{-1}$. Given the luminosity distance of PTF09axc, this translates to a flux density at $5 \mathrm{GHz}$ of $20 \mu \mathrm{Jy}$, a level that

\footnotetext{
11 Indeed, TDE disks may be so starved of magnetic flux that initial angular momentum transport may be dominated by exotic processes such as the Papaloizou-Pringle instability (Nealon et al. 2018) or fallback shocks (Chan et al. 2019), rather than the usual magnetorotational instability.
}

is detectable with current radio telescopes, although this flux estimate carries a substantial uncertainty.

If the soft X-ray spectra of X-ray-bright TDEs imply that those systems accrete in the equivalent of the XRB soft state, the fact that many TDEs have very weak or nonexistent earlytime radio emission is unsurprising (see Maccarone et al. 2003; van Velzen et al. 2013). We note that XMMSL1 J074008.2 -853927, another TDE with an X-ray power-law component (index $\Gamma=2$ ), was detected in radio (Alexander et al. 2017; Saxton et al. 2017), although XMMSL2 J144605.0+685735, which shows a power law with index $\Gamma=2.5$, was not (Saxton et al. 2019).

Finally, we note that our X-ray detections demonstrate that late-time TDE disks do not generally exhibit a different sort of state change: a collapse into a cold, gas pressuredominated state due to the development of a thermal instability. This type of collapse is predicted by simple applications of the popular $\alpha$-disk model but would imply that late-time TDE disks have luminosities far below what we observe (Shen \& Matzner 2014). Our observations further substantiate this point, which was recently made in the context of late-time detections of TDE disks in the FUV (van Velzen et al. 2019b). The evidence against very cold disks in (most) TDEs seen at late times could indicate that the nonlinear development of the thermal instability is suppressed by an iron opacity bump (Jiang et al. 2016) or, alternatively, magnetic pressure support (Begelman \& Pringle 2007; Sa̧dowski 2016; Jiang et al. 2019).

\subsection{Optical versus $X$-Ray-selected TDEs}

Many of the first TDE candidates were detected from their soft X-ray emission but either lacked contemporaneous searches for optical variability (Komossa \& Greiner 1999) or were observed not to show variable optical behavior (Greiner et al. 2000; Saxton et al. 2012, 2019). Later, optical and UV surveys discovered a second class of TDE candidates, which often possessed upper limits on their X-ray emission (Gezari et al. 2012; see also PTF09ge, ASASSN-14ae, and PTF09djl). More recently, a number of TDEs have been observed to exhibit both optical/UV and X-ray variability (e.g., Holoien et al. 2016a, 2016b; Wevers et al. 2019a). With such a diversity of X-ray $\left(L_{X}\right)$ and optical $\left(L_{\mathrm{opt}}\right)$ luminosities, it is fair to ask: do these transients all really stem from the same underlying type of event?

In the context of the reprocessing paradigm, this question has sometimes been answered (theoretically) in the affirmative by introducing a viewing angle dependence akin to the AGN unification model (Metzger \& Stone 2016; Dai et al. 2018; Lu \& Bonnerot 2020): edge-on TDEs obscure the X-rays from the inner accretion flow, but face-on TDEs are viewed through a low-density polar region and thus will be X-ray bright. Some observational characteristics, such as the emission lines in the optical part of the spectrum becoming more narrow as the TDE evolves, are consistent with this interpretation (Leloudas et al. 2019). The complicated three-dimensional geometry of the circularization/shock paradigm (Piran et al. 2015; Shiokawa et al. 2015) likely suggests a viewing angle dependence as well.

A different-possibly complementary-way to unify TDE candidates across a broad range of $L_{\mathrm{X}} / L_{\mathrm{opt}}$ ratios is to postulate a strong temporal dependence in $L_{\mathrm{X}} / L_{\mathrm{opt}}$. Our late-time detections of PTF09axc, PTF09ge, and ASASSN-14ae demonstrate that a substantial fraction of optically selected TDEs are 
$\mathrm{X}$-ray bright at late times, $\approx 5-10 \mathrm{yr}$ post-peak, signifying the presence of an exposed, compact accretion disk. If the optical emission is caused by circularization shocks, a delay between the optical and X-ray would be related to delays in forming the (inner, X-ray-emitting) accretion disk, as has been suggested by Shiokawa et al. (2015). If the optical is instead caused by reprocessing of the inner disk's X-rays and EUV, then an enshrouded inner disk will only become visible in X-rays after the reprocessing screen has diluted enough to permit an ionization breakout (Metzger \& Stone 2016; Roth et al. 2016).

Because the low $L_{X}$ values we observe are compatible with past X-ray nondetections (or, in the case of PTF09axc, its 2014 detection), we are unable to say whether this truly represents brightening of initially X-ray-dim TDEs. Deep limits on the $\mathrm{X}$-ray luminosity in several other optically selected TDEs suggest that brightening is certainly plausible (for references and limits, see the first paragraphs of the Discussion).

The nature of the X-ray light curve in optically selected TDEs is a crucial observable to constrain with future observations. The offset between the peaks of optical and $\mathrm{X}$-ray emission, $\Delta t_{\mathrm{o}-\mathrm{X}}$, is a key parameter for testing the idea of unification in time, rather than (or in addition to) angle. The distributions of $\Delta t_{\mathrm{o}-\mathrm{X}}$ will depend on the emission mechanism for the optical and X-ray light, as well as on event parameters such as $\beta, M_{.}$, and $\chi_{\text {. }}$

Depending on the delay between disruption and X-ray observation, an individual TDE could be in the equivalent of the soft X-ray spectral state or, as in the case of XMMSL2 J144605.0 +685735 , a hard power-law-like spectrum. ${ }^{12}$ We hypothesize that the X-ray-selected TDEs are, in this scenario, often discovered much longer after the disruption than are optically selected TDEs. This particular unification hypothesis would be falsified if observations months to years before the X-ray turn-on in a TDE candidate did not show signs of an optical enhancement. ${ }^{13}$

This scenario also implies that all optically selected TDEs will at some point emit X-ray radiation, as is true for three of the four sources we observed in this work. Sources that are detected in both optical and X-ray observations at early times (e.g., ASASSN-14li, ASASSN-15oi, and AT2018fyk; Holoien et al. 2016a, 2016b; Wevers et al. 2019a, respectively) could be explained in this scenario as sources with efficient circularization due, for instance, to high $\beta$, large $M$. (though this is disfavored by M. $-\sigma$ BH mass estimates), or large and retrograde SMBH spin.

The shape of the X-ray spectra, as well as the lower luminosities that we observed in PTF09axc, PTF09ge, and ASASSN-14ae, differ from the (soft) X-ray-discovered TDEs, which have soft thermal spectra and luminosities of order $L_{\mathrm{X}} \approx 10^{43-44} \mathrm{erg} \mathrm{s}^{-1}$ (Auchettl et al. 2017). This implies that our observed sources are, in this scenario, at an even later stage in the evolution of the mass fallback and accretion rate.

\subsection{Rates of Detection in Future X-Ray Surveys}

Near-future wide-field X-ray surveys are predicted to expand our sample of X-ray TDEs by 1-2 orders of magnitude. For example, the Einstein Probe is expected to find $\sim 100$ new TDEs per year (Yuan et al. 2015), while eROSITA is expected

\footnotetext{
12 A potential selection effect might be at play, as massive brightening of an $\mathrm{X}$-ray power law is more difficult to separate from AGN flares and thus will often not be classified as a TDE but rather as an AGN flare.

${ }^{13}$ In individual host galaxies, there could be reasons why the optical emission should be strongly reduced in these TDEs (such as the presence of a large amount of nuclear dust, e.g., Mattila et al. 2018).
}

to find $\sim 1000$ (Khabibullin et al. 2014). In this section, we revisit the latter estimate, making the following modifications to the model of Khabibullin et al. (2014).

1. We allow (in one of our models) the temperature at the inner edge of the accretion disk to be a function of SMBH spin.

2. We assume that the volumetric TDE rate is given by

$$
\dot{N}_{\mathrm{TDE}}=2.9 \times 10^{-5}\left(\frac{M .}{10^{8} M_{\odot}}\right)^{-0.4} \mathrm{yr}^{-1} \phi\left(M_{\bullet}\right) .
$$

This assumption takes a theoretical (per galaxy) TDE rate calibrated from observations of nearby galactic nuclei (Stone \& Metzger 2016) and multiplies it by $\phi\left(M_{\text {. }) \text {, the }}\right.$ $z=0.02$ black hole mass function from Shankar et al. (2009; their Table 3). ${ }^{14}$ We consider black hole masses between $10^{5}$ and $10^{8} M_{\odot}$ in our estimate. ${ }^{15}$ The volumetric TDE rate is $\sim 10^{-5} \mathrm{Mpc}^{-3} \mathrm{yr}^{-1}$ for this range.

3. We require the detection of at least 40 counts at a location where no source was previously detected by eROSITA.

We consider two different models for the TDE light curve and spectrum: (i) an optimistic theoretical model based on simple accretion disk theory and (ii) a more pessimistic quasiempirical model that is calibrated to reproduce the late-time X-ray properties of PTF09ge. In both cases, for simplicity, we only consider disruption of solar-type stars. The rates derived below are the X-ray detection rates, with no predictions about the fraction that is optically bright (see Section 3.2).

\subsubsection{Model I}

We assume that circularization occurs efficiently and that the mass accretion rate through the disk is

$$
\dot{M}_{\text {acc }}\left(M_{\bullet}, t\right)= \begin{cases}0 & t<t_{\text {fall }} \\ \dot{M}_{\max }\left(M_{\bullet}\right)\left[\frac{t}{t_{\text {fall }}\left(M_{\bullet}\right)}\right]^{-1.2} t \geqslant t_{\text {fall }}\end{cases}
$$

where $t_{\text {fall }}$ is the fallback time (Equation (2)). This power law is shallower than the canonical $t^{-5 / 3}$ decline of the mass fallback rate and is motivated by theoretical models for viscously spreading disks (Cannizzo et al. 1990), the late-time FUV light curves of TDEs (van Velzen et al. 2019b), and our own latetime X-ray detections. The maximum accretion rate $\dot{M}_{\max }$ is a factor of $\sim 3$ smaller than the peak fallback rate $\dot{M}_{\text {peak }}$. With this normalization, a total of half a solar mass of material is accreted.

The bolometric disk luminosity after one fallback time is

$$
\begin{aligned}
& L_{\mathrm{bol}}\left(t, M_{\bullet}, \chi_{\bullet}\right)=\min \left[L_{\mathrm{Edd}}\left(M_{\bullet}\right), \eta_{\bullet}\left(\chi_{\bullet}\right) \dot{M}_{\mathrm{acc}}(t) c^{2}\right] \\
& =\min \left[L_{\mathrm{Edd}}\left(M_{\bullet}\right),\right. \\
& \left.3 \times 10^{45}\left(\frac{\eta_{\bullet}\left(\chi_{\bullet}\right)}{0.057}\right)\left(\frac{M_{\bullet}}{10^{6} M_{\odot}}\right)^{-1 / 2}\left(\frac{t}{t_{\mathrm{fall}}}\right)^{-1.2} \mathrm{erg} \mathrm{s}^{-1}\right],
\end{aligned}
$$

\footnotetext{
14 The eROSITA instrument would be sensitive to TDEs with $z \lesssim 0.2$, and the Shankar et al. (2009) mass function varies little in this redshift range.

${ }^{15}$ Most of the TDEs are expected from the lower end in this mass range. Indeed, Wevers et al. (2017, 2019b), using and extrapolating the $M-\sigma$ correlation, showed that the masses of known TDEs favor the low-mass range.
} 
where $L_{\mathrm{Edd}}\left(M_{\bullet}\right)$ is the Eddington luminosity, and $\eta_{\bullet}$ is the standard radiative efficiency of a thin equatorial accretion disk $^{16}$. Here we have further assumed that the disk aligns itself into the SMBH equatorial plane after an initial period of misalignment. Typical alignment timescales are $\lesssim 100$ days for large $\left(\chi_{\bullet}>0.5\right)$ SMBH spins (Franchini et al. 2016), so alignment is a reasonable approximation for eROSITA observations, which have a typical cadence of 6 months. ${ }^{17}$ Equation (6) is close to the estimated bolometric luminosity of PTF09ge near peak $\left(\sim 8 \times 10^{44} \mathrm{erg} \mathrm{s}^{-1}\right.$, which is comparable to the Eddington limit for this source; see van Velzen et al. 2019b).

Equations (5) and (6) specify the bolometric luminosity, but here we are interested in soft X-ray observations of TDEs, and many optically selected TDEs (including three sources of our sample) have not been detected in X-rays at early times. Theoretically, TDEs may become X-ray bright when the central engine ionizes through a surrounding reprocessing layer (Metzger \& Stone 2016; Roth et al. 2016) or, if circularization is inefficient, after repeated shock interactions near stream apocenter (e.g., Dai et al. 2015; Shiokawa et al. 2015). The precise time when this occurs is uncertain. However, at least the early, super-Eddington phases of mass fallback are likely to be X-ray dim. ${ }^{18}$ If disk formation is inefficient, there is little accretion to produce X-rays (Shiokawa et al. 2015); even if disk formation is efficient, the inner disk can be heavily obscured by bound debris (Loeb \& Ulmer 1997; Coughlin \& Begelman 2014) or outflows (Miller 2015; Metzger \& Stone 2016; Dai et al. 2018; Lu \& Bonnerot 2020). However, as our present work shows, a large fraction of TDEs become $\mathrm{X}$-ray bright at later times, when the luminosity becomes subEddington. This occurs after

$$
t_{\text {Edd }}\left(M_{\bullet}, \chi_{\bullet}\right) \approx 1.5 \mathrm{yr}\left(\frac{M .}{10^{6} M_{\odot}}\right)^{-3 / 4}\left(\frac{\eta\left(\chi_{\bullet}\right)}{0.057}\right)^{5 / 6} .
$$

Here $t_{\mathrm{Edd}}$ is the time after which the accretion rate becomes sub-Eddington. In practice, we consider a TDE at redshift $z$ to be detectable by eROSITA after $t_{\mathrm{Edd}}\left(M_{\bullet}, \chi_{\bullet}\right)$, as long as

$$
\frac{L}{4 \pi d_{L}^{2}(z) K(z)} \geqslant f_{\text {lim }}
$$

where $d_{L}(z)$ is the luminosity distance and

$$
\begin{gathered}
f_{\lim }=\frac{C_{\text {crit }}}{t_{\text {int }} \int_{\nu_{\min }}^{\nu_{\max }} \frac{S_{\nu}(\nu) A(\nu) e^{-\xi(\nu)}}{h \nu} d \nu} \\
K(z)^{-1}=\frac{(1+z) \int_{\nu_{\min }}^{\nu_{\max }} \frac{S_{\nu}(\nu(1+z)) A(\nu) e^{-\xi(\nu)}}{h \nu} d \nu}{\int_{\nu_{\min }}^{\nu_{\max }} \frac{S_{\nu}(\nu) A(\nu) e^{-\xi(\nu)}}{h \nu} d \nu} .
\end{gathered}
$$

Here $C_{\text {crit }}$ and $t_{\text {int }}$ are the minimum number of counts resulting in a detection and the integration time, respectively (which we

\footnotetext{
16 The efficiency $\eta$. ranges from 0.038 to 0.42 as $a$. goes from -1 to 1 and is computed as in Bardeen et al. (1972).

17 In principle, alignment can take longer than 6 months if $\chi_{\bullet} \lesssim 0.5$, but $\eta_{\bullet}$ is considerably less sensitive to SMBH spin in this regime.

18 At least for most viewing angles, observers aligned with the poles may see X-ray emission from a jet according to the unification model of Dai et al. (2018).
}

take to be 40 and $240 \mathrm{~s}$ following Khabibullin et al. 2014), $A_{\nu}$ is the effective area as a function of energy, ${ }^{19} e^{-\xi(\nu)}$ accounts for photoelectric absorption, ${ }^{20}$ and $S_{\nu}$ is the SED, which we take to be a blackbody with an effective temperature corresponding to the temperature near the ISCO, as given by Equation (9) of Lodato \& Rossi (2011). ${ }^{21}$ We integrate SEDs between $h \nu_{\min }=0.2 \mathrm{keV}$ and $h \nu_{\max }=2 \mathrm{keV}$ (following Khabibullin et al. 2014).

The total number of new TDEs detected every year is

$$
\begin{aligned}
N_{\mathrm{det}} & =\int_{0}^{1 \mathrm{yr}} \int_{M_{\min }}^{M_{\max }} \int_{0}^{z \lim \left(M_{\bullet}\right)} \frac{d N}{d t d M . d z} d z d M . d t \\
& =\int_{0}^{1 \mathrm{yr}} \int_{M_{\min }}^{M_{\max }} \int_{0}^{\left.V_{\mathrm{c}} z_{\lim }\left(M_{.}\right)\right]} \dot{N}_{\mathrm{TDE}} d V_{\mathrm{c}}(z) d M . d t,
\end{aligned}
$$

where $d N / d t d M . d z$ is the differential TDE rate per unit SMBH mass per unit redshift, and $z_{\lim }$ is the maximum redshift to which a TDE in a given mass bin could be detected. In the second line, $\dot{N}_{\mathrm{TDE}}$ is the volumetric TDE rate (Equation (4)), while $d V_{c}$ is the comoving volume element. Conservatively, $z_{\lim }$ satisfies

$$
\begin{aligned}
& \frac{L\left(t_{o}+6 \text { months }\right)}{4 \pi d_{L}\left(z_{\lim }\right)^{2} K\left(z_{\lim }\right)}=f_{\lim } \\
& t_{o}=\max \left[t_{\text {edd }}\left(M_{\bullet}, \chi_{\bullet}\right), t_{\text {fall }}\right],
\end{aligned}
$$

where $t_{o}$ is when the $\mathrm{X}$-rays turn on and 6 months is the time it takes eROSITA to scan the entire sky.

The top panel of Figure 2 shows the eROSITA detection rate as a function of SMBH mass and spin, assuming that all TDE hosts have the same mass and spin combination and the total TDE rate is $10^{-5} \mathrm{Mpc}^{-3} \mathrm{yr}^{-1}$. For a flux-limited sample of TDEs produced by rapidly spinning black holes, there are 1-2 orders of magnitude more detections when the black hole spin is universally prograde (with respect to the accretion disk's rotation) than universally retrograde, irrespective of the SMBH mass bin we consider. In stark contrast to optically selected TDE samples (Section 3.4), an X-ray-selected sample will be strongly biased toward prograde black hole spins, though this bias abates if the SMBH spin distribution is very bottom-heavy (with typical $\chi_{\bullet} \ll 1$ ).

In the bottom panel of Figure 2, we use the more realistic, nonuniform (in SMBH mass) TDE rate given by Equation (4). Smaller SMBH masses are strongly favored in flux-limited $\mathrm{X}$-ray TDE samples because (i) their disks have higher effective temperatures, increasing the luminosity in the eROSITA band; (ii) they preferentially occur in denser and cuspier galactic nuclei, where two-body relaxation times are shorter and TDE rates are higher; and (iii) such SMBHs are more common, given our assumed mass function. Our predictions are closest to those of Khabibullin et al. (2014) when we set $\chi_{\bullet} \approx 0.9-0.95$, where the effective temperature at the inner disk edge in our model matches theirs. The observed black hole mass distribution of soft X-ray-selected TDEs (Wevers et al. 2019b) does not show evidence for a larger

\footnotetext{
19 https://wiki.mpe.mpg.de/eRosita/erocalib_calibration

${ }^{20}$ This is derived from the XSPEC PHABS multiplicative model with $N_{\mathrm{H}}=5 \times 10^{20} \mathrm{~cm}^{-2}$ following Khabibullin et al. (2014).

${ }^{21}$ The effective temperature actually goes to zero at the ISCO in this model. In practice, we evaluate $T_{\text {eff,in }}$ at 1.36 times the ISCO, where the effective temperature is maximized.
} 

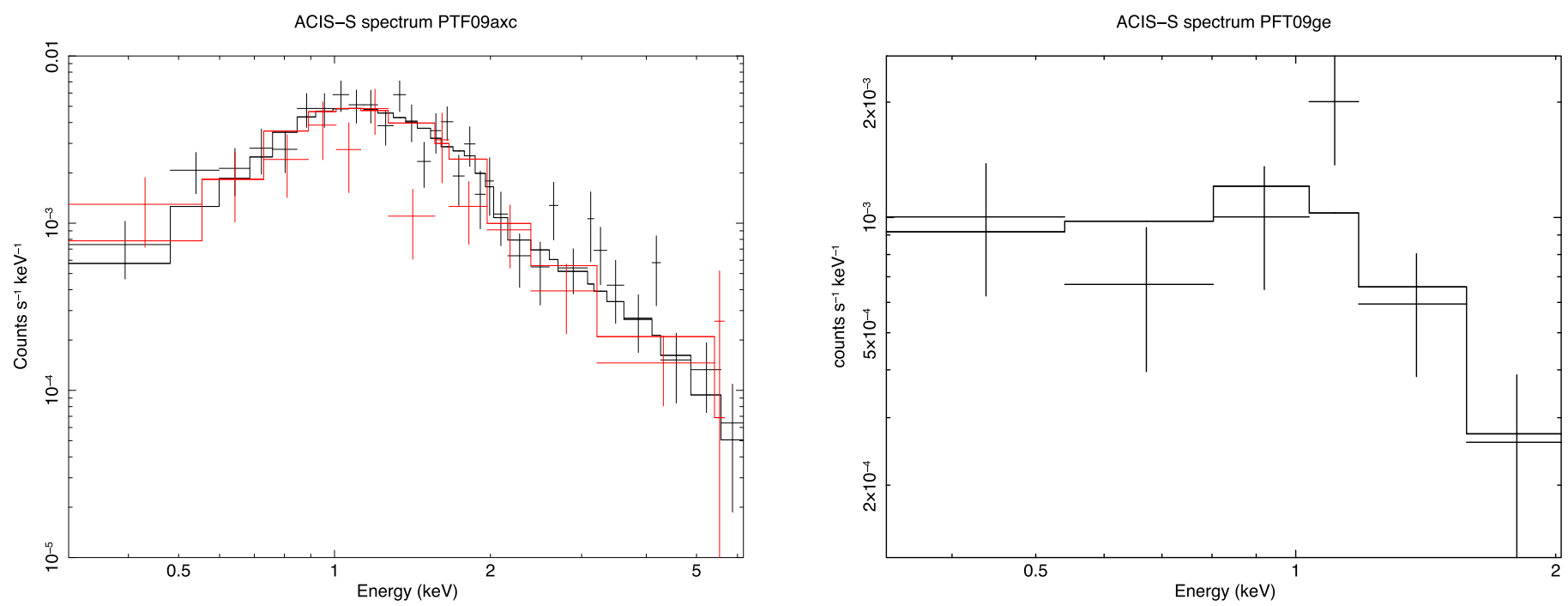

Figure 1. Left panel: Chandra ACIS-S spectrum of PTF09axc fitted with a power law folded through the detector response. The black data points are from ObsID 19532, and the red (/gray) data crosses are from ObsID 20879. The best-fit power-law index is $2.5 \pm 0.1$. Right panel: Chandra ACIS-S spectrum of PTF09ge fitted with a power law. The best-fit power-law index is $3.9 \pm 0.4$.

number of TDEs from smaller SMBH masses, although there is a hint for this in hard X-ray-selected TDEs.

Table 4 shows the mass-integrated eROSITA detection for a few different SMBH spin parameters (assuming equal intrinsic numbers of prograde and retrograde disruptions). The detection rate is a strong function of the uncertain SMBH spin distribution: in our fiducial model (where the SMBH mass function extends down to $M_{\bullet}=10^{5} M_{\odot}$ ), we predict $\approx 1000$ detections $\mathrm{yr}^{-1}$ for $\chi_{\bullet}=0.99$ but only $170 \mathrm{yr}^{-1}$ for $\chi_{\bullet}=0$. For large values of $\chi_{\bullet}$, a flux-limited sample is strongly dominated by the $50 \%$ of TDE disks we assume to align into prograde equatorial configurations; depending on the combination of $M$. and $\chi_{\bullet}$, this "prograde bias" can range from $\sim 10 \%$ to multiple orders of magnitude. Prograde disks and high values of $\left|\chi_{.}\right|$are favored because of their higher bolometric luminosities and effective temperatures.

The X-ray discovery rate is dominated by the smallest $\left(M_{\bullet} \sim 10^{5} M_{\odot}\right)$ SMBHs, a part of parameter space where the SMBH occupation fraction is poorly constrained. Interestingly, a flux-limited and X-ray-selected TDE sample can be a more sensitive probe of the bottom end of the SMBH mass function than a volume-complete TDE sample would be. ${ }^{22}$ In our models, this is true for $\chi \bullet \lesssim 0.9$ and is due to the fact that (unless most SMBHs are nearly extremal) $\mathrm{X}$-ray emission is typically on the Wien tail of TDE disks and is thus highly sensitive to populations of smaller SMBHs. Furthermore, the eROSITA TDE detection rate may also be a strong indicator of the SMBH spin distribution, even if the spins of individual TDE-hosting SMBHs cannot be measured. This is analogous to the manner in which statistical samples of TDEs may probe the SMBH spin distribution near the Hills mass (Kesden 2012), although it is not limited to the largest TDE hosts.

Many TDE light curves would be reasonably well-sampled with eROSITA. For example, for a $10^{5}\left(10^{6}\right) M_{\odot} \mathrm{SMBH}$ with a spin of 0.99 , prograde disruptions would be visible, on average, for 27 (5.3) yr. This would give an average of eight detections

\footnotetext{
${ }^{22}$ Using Equation (4), we find that reducing $M_{\min }$ from $10^{6}$ to $10^{5} M_{\odot}$ increases the volumetric TDE rate by a factor of $\approx 8.5$.
}

per TDE, considering the cadence and nominal duration of the eROSITA all-sky survey (6 months and $4 \mathrm{yr}$, respectively).

\subsubsection{Model II}

Next, we reestimate eROSITA detection rates with a quasiempirical model calibrated to reproduce the observed properties of PTF09ge. While the model from the prior section was arguably an optimistic one (in its assumption that all TDEs will become X-ray bright after the disk accretion rate becomes subEddington), this empirically calibrated model can be viewed as a rather pessimistic scenario, where we impose a long, adjustable period of X-ray darkness on most TDEs. In this model, the bolometric luminosity is

$$
\begin{aligned}
& L_{\mathrm{bol}}=\left\{\begin{array}{l}
0, \\
\min \left[L_{\mathrm{Edd}}\left(M_{\bullet}\right), 2.5 \times 10^{43} \mathrm{erg} \mathrm{s}^{-1}\right. \\
\left.\left(\frac{t}{t_{\text {fall }}}\right)^{-1.2}\left(\frac{M .}{3 \times 10^{6} M_{\odot}}\right)^{-1 / 2}\right], \quad t \geqslant t_{\mathrm{o}}
\end{array},\right. \\
& t_{o}=\max \left[t_{\mathrm{br}}, t_{\mathrm{Edd}}, t_{\text {fall }}\right] .
\end{aligned}
$$

This reproduces the inferred late-time bolometric luminosity for PTF09ge ${ }^{23}$ for its inferred SMBH mass of $\sim 3 \times 10^{6} M_{\odot}$ (Wevers et al. 2019b). The scalings with SMBH mass and time are the same as in the theoretical model, but SMBH spin is not explicitly included. The X-rays turn on after the brightening time $\left(t_{\mathrm{br}}\right)$, as long as this is greater than the fallback time and the luminosity is sub-Eddington.

We assume, based on our late-time observations of PTF09ge, that the spectrum is a blackbody with an effective temperature $k T=0.18 \mathrm{keV}$. The bolometric luminosity is up to 2 orders of magnitude smaller than in model I, which would reduce the detection rate. However, this is partially compensated for by the harder spectrum.

\footnotetext{
$\overline{{ }^{23}}$ Here $2.7 \times 10^{41} \mathrm{erg} \mathrm{s}^{-1}$ is derived from the best-fit blackbody spectrum for this event.
} 

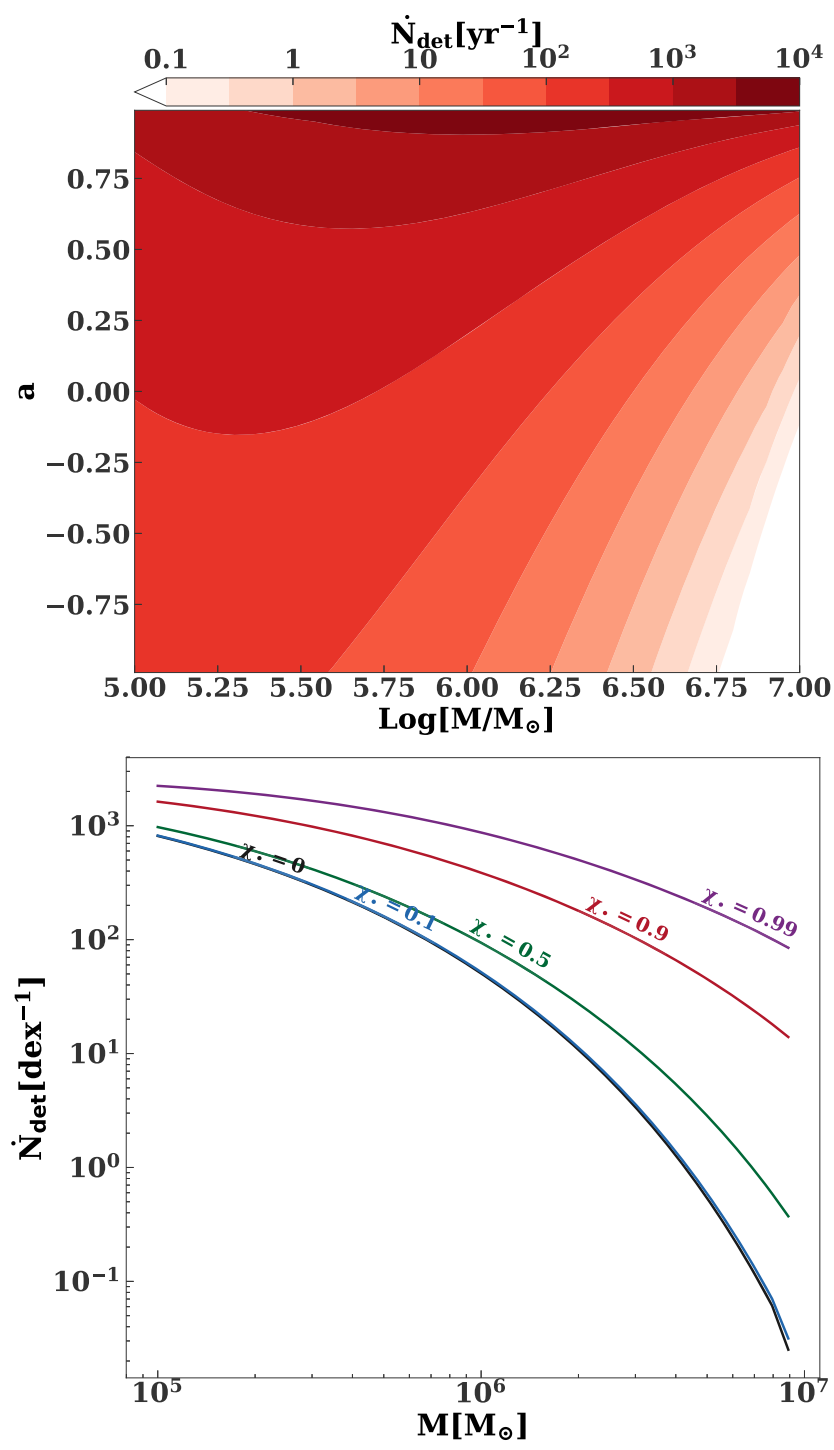

Figure 2. Top panel: rate of eROSITA detections as a function of SMBH mass and spin for a fixed TDE rate of $10^{-5} \mathrm{Mpc}^{-3} \mathrm{yr}^{-1}$. These are the detection rates assuming that TDEs are distributed as a delta function with a specific mass and spin (i.e., each SMBH mass and spin is assumed equally likely). Bottom panel: rate of eROSITA detections as a function of SMBH mass for a selection of spin parameters. These lines are a convolution of the rates from the top panel with the SMBH mass function and a theoretical estimate of TDE rates as a function of $M$. (Equation (4)). We assume that $50 \%$ of TDE disks align into prograde equatorial and $50 \%$ align into retrograde equatorial configurations by the time of observation; see the discussion in Section 3.4.

Table 5 shows the mass-integrated eROSITA detection rates for two different brightening times $t_{\mathrm{br}}$. For higher SMBH minimum masses $\left(M_{\min }=10^{6} M_{\odot}\right)$ and small brightening times, the rates are comparable to the estimates from model I for moderate spins (with $0.5 \lesssim \chi_{\bullet} \lesssim 0.9$; see Table 4). However, the predicted rates for lower SMBH mass limits $\left(M_{\min }=10^{5} M_{\odot}\right)$ and/or larger brightening times are smaller than the zero-spin case in model I.

Taking into account that eROSITA will require the detection of at least 40 counts, whereas on a previous pass, eROSITA did not detect the source, in model II, the TDEs are, on average, observable for 2 (6) yr after detection for a brightening time of 1 (5) yr. This implies significantly poorer temporal sampling in eROSITA light curves than in model I.

\subsection{Retrograde and Prograde TDE Disks}

In the previous section, we saw that X-ray-selected TDE samples are likely to possess a strong bias toward prograde configurations of SMBH spin $\chi_{\text {. }}$ and disk angular momentum $\boldsymbol{L}_{d}$ (i.e., $\chi_{\bullet} \cdot \boldsymbol{L}_{d}>0$ ), as long as typical SMBH spin magnitudes are reasonably large $\left(\chi_{\bullet} \gtrsim 0.5\right)$. In this section, we discuss the prospects for observing this prograde preference and build simple toy models to show how it contrasts with the likely weaker orientation biases in optically selected TDE samples, which may even exhibit a preference for retrograde configurations $\left(\chi_{\bullet} \cdot \boldsymbol{L}_{d}<0\right)$.

Our observations of PTF09ge and the observations presented in the literature for IC 3599 indicate that soft X-ray emission may remain visible for roughly a decade after the peak of a tidal disruption flare, although the majority of X-ray observations of TDEs done $>8 \mathrm{yr}$ after discovery find that they have transitioned to a nonthermal state with power-law spectral shapes where the power-law index is $\approx 2.5$. Nevertheless, for those sources with a soft spectrum years after the initial discovery, one could use such late-time TDE observations to directly measure $\mathrm{SMBH}$ spin through continuum fitting techniques. While continuum fitting is a fruitful method of measuring the spins of stellar mass black holes in XRBs (McClintock et al. 2014), it has only rarely been applied to SMBHs because (i) AGNs typically produce dusty tori, and these complicate the X-ray spectral fitting, and (ii) the spectral peak of a quasi-thermal AGN disk is usually in observationally inaccessible EUV bands.

The relatively cool temperatures of TDE disks (in contrast to those of XRBs) mean that quasi-thermal soft X-rays will generally be on the Wien tail of emission (Lodato \& Rossi 2011), and their production will be dominated by the innermost gas annuli of the disk. As a result, quasi-thermal $\mathrm{X}$-rays from TDEs will be exponentially sensitive to the size of the disk inner edge and therefore will depend strongly on $\mathrm{SMBH}$ spin. At early times, the disk inner edge is nontrivial to estimate. Because two-body scattering feeds stars to SMBHs from a roughly isotropic distribution of angles, TDE disks are generically born with order unity tilts. A tilted thin disk will be truncated near the innermost stable spherical orbit (ISSO), but the high early-time accretion rates of TDEs may cause their innermost disk annuli to extend inside the ISSO. ${ }^{24} \mathrm{~A}$ greater problem at early times, however, is the messy hydrodynamical environment of the disk: if the stellar pericenter was sufficiently nonrelativistic $\left(R_{\mathrm{p}} \gg R_{\mathrm{g}}\right)$, the disk may retain substantial eccentricity (Shiokawa et al. 2015), and if optically thick stellar debris subtends a large solid angle on the sky, the majority of the X-ray flux may be absorbed in a reprocessing layer (Guillochon et al. 2014; Metzger \& Stone 2016).

At late times, however, accretion rates will have dropped to sub-Eddington levels, shifting the disk inner edge close to the test particle value; many fallback times will have passed, enabling more complete circularization (Hayasaki et al. 2016; Bonnerot et al. 2017); reprocessing layers will have dissipated, revealing the inner disk (Metzger \& Stone 2016; van Velzen et al. 2019b); and internal torques will have had time to align the TDE disk angular momentum vector with the black hole spin vector (Franchini et al. 2016). Thus, if we interpret the soft

\footnotetext{
${ }^{24}$ For example, with accretion disks tilted with respect to the black hole spin by an angle of $15^{\circ}$ and a thickness of the order of 0.2 , the simulations of Fragile (2009) found the inner edge to be nearly independent of spin.
} 
Table 4

Estimated eROSITA TDE Detection Rates

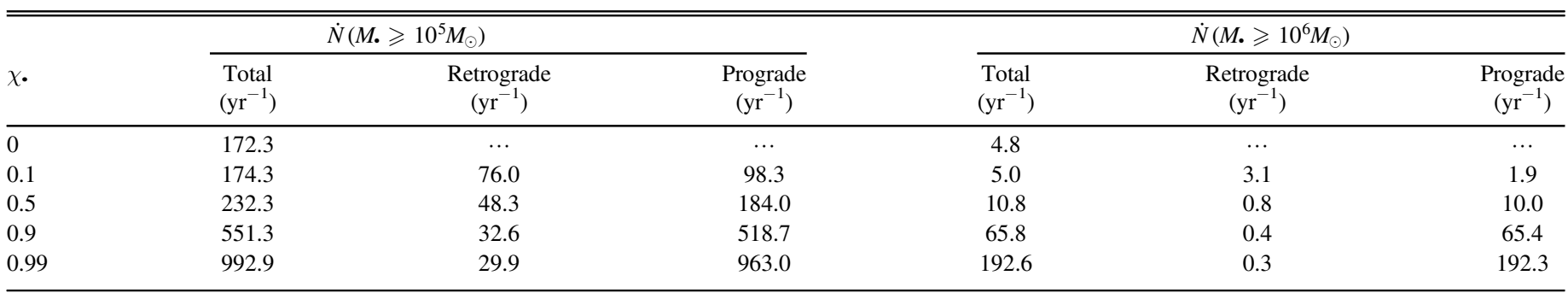

Note. Estimated eROSITA TDE detection rates using the formalism outlined in Section 3.3.1. Column (1) is the SMBH spin. Columns (2)-(4) give the total, retrograde, and prograde detection rates including all SMBHs between $10^{5} M_{\odot}$ and the Hills mass. Columns (5)-(7) give the total, retrograde, and prograde detection rate including SMBHs with masses between $10^{6}$ and the Hills mass. In all cases, we assumed an equal intrinsic number of prograde and retrograde disruptions (see the discussion in Section 3.4). For these estimates, we assume the TDE mass function from Equation (4) but discard TDEs with Galactic latitudes $\leqslant 30^{\circ}$, as in Khabibullin et al. (2014).

X-ray spectrum of PTF09ge, and perhaps ASASSN-14ae, as quasi-thermal, it is reasonable to expect thin disks in the SMBH equatorial plane, with inner edges at the test particle ISCO. We may now ask the question: do we expect an imbalance in the number of prograde and retrograde TDEs? For a volumecomplete sample, the answer is clearly no. However, for a more practical, flux-limited sample of TDEs, there are strong reasons to suspect an imbalance. We have already predicted that fluxlimited, X-ray-selected TDE samples can exhibit an enormous prograde bias (e.g., Table 4). In this section, we investigate whether the same bias should be apparent for a flux-limited but optically selected TDE sample. While the origin of TDE optical emission remains contested between "shock-powered" and "reprocessing" models, both of these scenarios have peak luminosities that will depend strongly on the orbital precession of debris streams and therefore on SMBH spin.

To leading post-Newtonian (PN) order, both apsidal precession (precession of the debris stream's Runge-Lenz vector within the orbital plane) and nodal precession (precession of the orbital plane's angular momentum vector about the SMBH spin vector) are larger for retrograde than for prograde orbits (Merritt et al. 2010). Neglecting for now the possibility that extreme nodal precession may prevent stream selfintersections, ${ }^{25}$ the greater apsidal shifts for debris from retrograde TDEs means that these debris streams will selfintersect and dissipate energy at smaller radii. Smaller stream self-intersection radii $R_{\mathrm{SI}}$ will probably yield higher peak optical luminosities, regardless of the dominant optical power source in observed TDEs. In the "reprocessing paradigm," smaller $R_{\mathrm{SI}}$ will mean faster disk formation and higher peak accretion rates $\dot{M}$ onto the SMBH, although this must be weighed against the potentially greater radiative efficiency of prograde disks. In the "circularization paradigm," smaller $R_{\mathrm{SI}}$ values will thermalize greater amounts of bulk kinetic energy.

The translation between the self-intersection radius $R_{\mathrm{SI}}$ and optical luminosity is currently an unsolved problem. Under the assumption that most of the observed optical emission is shock-

\footnotetext{
25 Tidal disruptions of stars in the relativistic regime (e.g., white dwarfs disrupted by intermediate-mass black holes or solar mass main-sequence stars disrupted by a black hole with $M_{\bullet}=10^{7-8} M_{\odot}$ ) around spinning SMBHs may lead to stellar debris streams that fail to promptly self-interact, unless the inclination of the stellar orbit is nearly perpendicular to the $\mathrm{BH}$ spin axis or the thickness of the debris streams is large enough that they always intersect (Dai et al. 2013; Guillochon \& Ramirez-Ruiz 2015; Hayasaki et al. 2016).
}

Table 5

Estimated TDE Detection Rates with eROSITA Using the Quasi-empirical Model Outlined in Section 3.3.2

\begin{tabular}{lcc}
\hline \hline $\begin{array}{l}t_{\mathrm{br}} \\
(\mathrm{yr})\end{array}$ & $\dot{N}\left(M . \geqslant 10^{5} M_{\odot}\right)$ & $\begin{array}{c}\dot{N}\left(M . \geqslant 10^{6} M_{\odot}\right) \\
\left(\mathrm{yr}^{-1}\right)\end{array}$ \\
\hline 1 & 150 & 24 \\
5 & 16 & 2.6 \\
\hline
\end{tabular}

powered, we will use the following toy model for peak luminosity:

$$
L_{\text {peak }}=\eta_{\mathrm{SI}} \frac{G M \cdot \dot{M}_{\text {peak }}}{R_{\mathrm{SI}}}
$$

where, as before, $\dot{M}_{\text {peak }}$ is the peak mass fallback rate. The dimensionless number $\eta_{\mathrm{SI}} \leqslant 1$ is the fraction of stream kinetic energy thermalized and radiated at the self-intersection; for simplicity, we take it to be a constant. ${ }^{26}$ A flux-limited survey will find a differential number of TDEs per bin of pericenter $R_{\mathrm{p}}$ and inclination $i$ that scales as $d N_{\text {det }} / d i d R_{\mathrm{p}} \propto$ $L_{\text {peak }}^{3 / 2}\left(i, R_{\mathrm{p}}\right)\left(d \dot{n} / d i d R_{\mathrm{p}}\right)$, where the differential rate $d \dot{n} / d i d R_{\mathrm{p}} \propto$ $\sin i$ if we are in the full loss cone (FLC) regime, and $d \dot{n} / d i d R_{\mathrm{p}} \propto \sin i \times \delta\left(R_{\mathrm{p}}-R_{\mathrm{t}}\right)$ if we are in the empty loss cone (ELC) regime (we have assumed isotropy in stellar arrival directions in both regimes).

The dependence of $L_{\text {peak }}$ on $i$ and $R_{\mathrm{p}}$ can be computed by defining the self-intersection radius (Dai et al. 2015),

$$
R_{\mathrm{SI}}=\frac{R_{\mathrm{p}}(1+e)}{1+e \cos (\pi+\delta \omega / 2)},
$$

where $e$ is the eccentricity of the elliptical orbit of the stream of material formed by the disrupted star. For convenience, we take the eccentricity of the most tightly bound debris, $e_{\min }=$ $1-2\left(M_{\star} / M_{\bullet}\right)^{1 / 3} / \beta$. Here we have made use of the per-orbit apsidal shift angle, $\delta \omega$, which, to lowest PN order in

\footnotetext{
26 As Lu \& Bonnerot (2020) have noted, a large fraction of the thermalized stream kinetic energy may be lost to adiabatic degradation prior to the time it can be emitted. Because the fractional energy loss to $P d V$ work depends on gas optical depth at $R_{\mathrm{SI}}$ and therefore on $M$. and other parameters, the assumption of constant $\eta_{\mathrm{SI}}$ is crude. Deriving a more complete theoretical model is, however, beyond the scope of this work.
} 
dimensionless SMBH spin, $\chi_{\bullet}$, is (Merritt et al. 2010)

$$
\delta \omega=A_{\mathrm{S}}-2 A_{\mathrm{J}} \cos i
$$

where

$$
\begin{gathered}
A_{\mathrm{S}}=\frac{6 \pi}{c^{2}}\left(\frac{G M \cdot}{R_{\mathrm{p}}(1+e)}\right), \\
A_{\mathrm{J}}=\frac{4 \pi \chi_{\bullet}}{c^{3}}\left(\frac{G M \cdot}{R_{\mathrm{p}}(1+e)}\right)^{3 / 2} .
\end{gathered}
$$

In the ELC regime, for fixed SMBH mass and stellar properties, the retrograde fraction is simply

$$
f_{\mathrm{ret}}^{\mathrm{ELC}}=\frac{\int_{\pi / 2}^{\pi} \sin i[1+e \cos (\pi+\delta \omega / 2)]^{3 / 2} d i}{\int_{0}^{\pi} \sin i[1+e \cos (\pi+\delta \omega / 2)]^{3 / 2} d i} .
$$

In the FLC regime, a second integral is necessary:

$$
f_{\mathrm{ret}}^{\mathrm{FLC}}=\frac{\int_{\pi / 2}^{\pi} \sin i \int_{R_{\min }}^{R_{\mathrm{t}}} R_{\mathrm{p}}^{-3 / 2}[1+e \cos (\pi+\delta \omega / 2)]^{3 / 2} d R_{\mathrm{p}} d i}{\int_{0}^{\pi} \sin i \int_{R_{\min }}^{R_{\mathrm{t}}} R_{\mathrm{p}}^{-3 / 2}[1+e \cos (\pi+\delta \omega / 2)]^{3 / 2} d R_{\mathrm{p}} d i} .
$$

Here $R_{\mathrm{p}}$ ranges from a maximum value of $R_{\mathrm{t}}$ down to a minimum value of $R_{\min }\left(\chi_{\bullet}, i\right)$. This minimum value, the innermost bound spherical orbit (IBSO), is computed from the Kerr geodesic equations (Bardeen et al. 1972). The IBSO is larger for retrograde spins, which (via Equation (13)) introduces a prograde bias.

We illustrate the overall orientation bias in flux-limited samples of shock-powered TDEs in Figure 3. There is no bias when $\chi_{\bullet}=0$ (as symmetry demands), but the bias becomes notable when $\chi \cdot \gtrsim 0.5$. Interestingly, the bias is qualitatively different in the two regimes of loss cone repopulation. In the ELC regime, there is almost no bias for $M_{\bullet} . \lesssim 10^{6} M_{\odot}$, but there is a moderate retrograde bias for larger SMBHs. In the FLC regime, there is a moderate prograde bias across all bins of $M$. Since the ELC regime predominates for high-mass SMBHs and the FLC regime predominates for low-mass SMBHs (Stone \& Metzger 2016), we expect that flux-limited, shock-powered TDE samples will exhibit a prograde bias for $M_{\bullet} \lesssim 10^{6.5} M_{\odot}$ and a retrograde bias at higher masses.

We may also consider a similar sort of toy model for the reprocessing picture of TDE optical luminosity, designed to illustrate the competition between disk formation (which is faster for retrograde orbits) and the radiative efficiency of a circularized accretion disk (which is higher for prograde orbits). Let us say that the peak optical luminosity in a reprocessing model is

$$
L_{\text {peak }}=\eta_{\bullet} \eta_{\mathrm{r}} \dot{M}_{\max } c^{2},
$$

where $\eta_{\bullet}$ is the standard radiative efficiency of a thin equatorial accretion disk (see Section 3.3), and the efficiency with which an optically thick reprocessing layer converts X-ray and extreme UV photons to optical emission is assumed (again, for simplicity) to be a constant, $\eta_{\mathrm{r}}$. Here $\dot{M}_{\max }$ does not represent the peak mass fallback rate to pericenter, $\dot{M}_{\max }=$ $\frac{M_{\star}}{3}\left(t / t_{\text {fall }}\right)^{-5 / 3}$, but rather the peak accretion rate through the

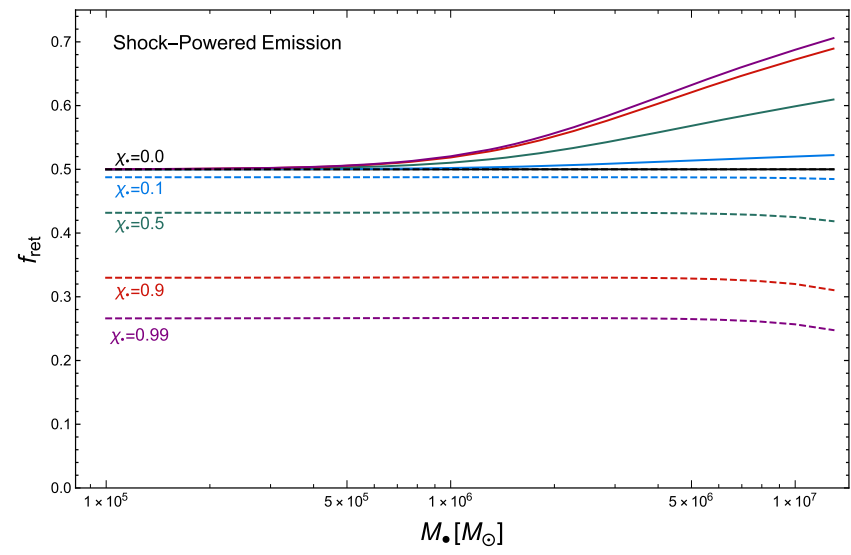

Figure 3. Fraction of TDEs with retrograde disks, $f_{\text {ret }}$, in a flux-limited sample of (i) optically selected and (ii) shock-powered tidal disruption flares. In the ELC regime (solid lines), there is no preference for retrograde orbits when

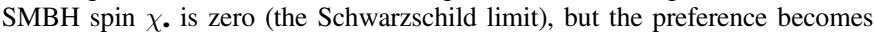
more notable for higher values of $\chi$. (shown and labeled as color-coded curves). Conversely, in the FLC regime (dashed lines), the preference is for prograde disks.

disk, which we parameterize as

$$
\dot{M}_{\max }=\frac{M_{\star}}{2 t_{\text {circ }}},
$$

where we assume that the "circularization timescale," $t_{\text {circ }}$, is a function only of the self-intersection radius and is related to the fallback time for the most tightly bound debris as $t_{\text {circ }}=$ $t_{\text {fall }}\left(R_{\mathrm{SI}} / R_{\mathrm{g}}\right)^{\xi}$. This power-law parameterization of the disk formation timescale is crude but will suffice to explore what types of disk orientation biases we expect if reprocessing is responsible for the observed optical emission. We find modified versions of the ELC and FLC regime retrograde fractions:

$$
\tilde{f}_{\text {ret }}^{\mathrm{ELC}}=\frac{\int_{\pi / 2}^{\pi} \sin i\left[1+e \cos \left(\pi+\frac{\delta \omega}{2}\right)\right]^{3 \xi / 2} \eta_{\bullet}^{3 / 2} d i}{\int_{0}^{\pi} \sin i[1+e \cos (\pi+\delta \omega / 2)]^{3 \xi / 2} \eta_{\bullet}^{3 / 2} d i},
$$

and

$$
=\frac{\int_{\pi / 2}^{\pi} \sin i \int_{R_{\min }}^{R_{\mathrm{t}}}\left(\frac{\eta_{\bullet}}{R_{\mathrm{p}}^{\xi}}\right)^{3 / 2}\left[1+e \cos \left(\pi+\frac{\delta \omega}{2}\right)\right]^{3 \xi / 2} d R_{\mathrm{p}} d i}{\int_{0}^{\pi} \sin i \int_{R_{\min }}^{R_{\mathrm{t}}}\left(\frac{\eta_{\bullet}}{R_{\mathrm{p}}^{\xi}}\right)^{3 / 2}\left[1+e \cos \left(\pi+\frac{\delta \omega}{2}\right)\right]^{3 \xi / 2} d R_{\mathrm{p}} d i} .
$$

We illustrate the retrograde fractions in a flux-limited, reprocessing-powered TDE sample in Figure 4. In contrast to our earlier shock-powered calculations, our toy model for reprocessing power almost always exhibits a prograde disk bias, as this configuration yields much higher radiative efficiencies. The detailed nature of the orientation bias depends on the power-law index $\xi$ encoding the dependence of circularization efficiency on $R_{\mathrm{SI}}$ (in this figure, we use $\chi=0.5)$. Very high values of $\xi(\gtrsim 2)$ can create a retrograde bias in a sample of TDEs in the ELC regime, but this level of sensitivity to $R_{\mathrm{SI}}$ is not suggested by existing hydrodynamical 


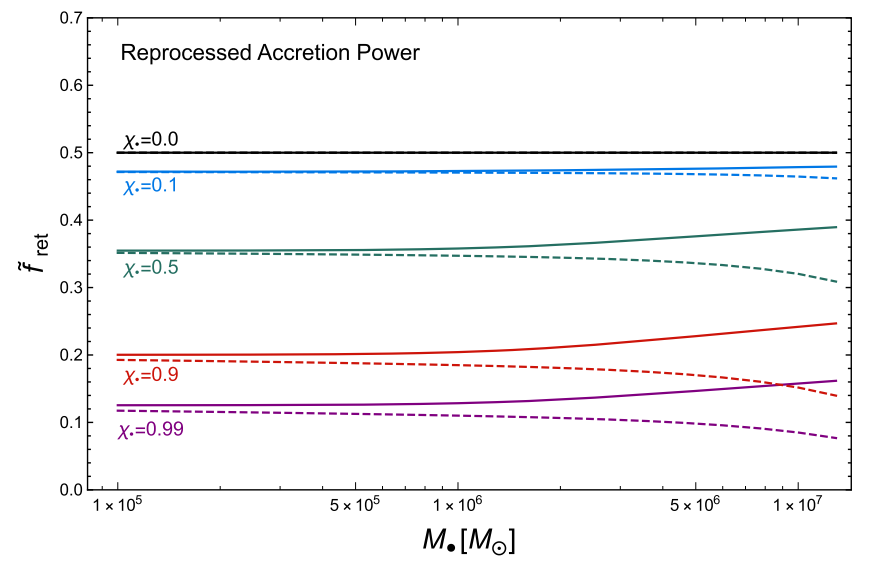

Figure 4. Same as Figure 3, but we now compute the retrograde fraction $\left(\tilde{f}_{\text {ret }}\right)$ considering a model for optical emission based on reprocessed X-ray/EUV emission from an inner accretion disk. In contrast to the shock-powered model of Figure 3, reprocessing-powered TDEs almost always show a bias for prograde disks due to radiative efficiency considerations. This bias is generally strongest for the ELC regime and smaller SMBHs but depends somewhat on the power-law index $\xi$ (assumed to be 0.5 in this plot). If $\xi \gtrsim 2$, a weak retrograde bias may be recovered in the ELC regime.

simulations of circularization (Bonnerot et al. 2016; Hayasaki et al. 2016). The overall level of bias depends on $\chi$. but shows little variation with $M$.

Of the TDEs we have observed, both PTF09ge and ASASSN-14ae have late-time accretion disks whose FUV properties were modeled in van Velzen et al. (2019b). Our X-ray detections are compatible with these disk models, provided the disk in PTF09ge is prograde with respect to a very rapidly spinning SMBH, and the disk in ASASSN-14ae is retrograde with respect to a spinning $\mathrm{SMBH}$. While this sample is too small (and our disk models so far too crude) to meaningfully constrain $f_{\text {ret }}$, these observations, and the arguments in this section, highlight the potential of future late-time observations and modeling to determine the dependence of peak flare luminosity on the inclination of the disrupted star's orbit. This may also serve as a useful test between different models of optical power sources in TDEs, as it would be hard to explain a pronounced retrograde bias in the reprocessing paradigm.

In this section, we have used simple but illustrative optical emission models to demonstrate that the selection effects operating in flux-limited, optically selected TDE samples favor

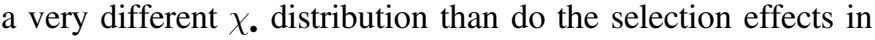
flux-limited, X-ray-selected samples (Section 3.3). Specifically, an X-ray-selected sample will be biased strongly toward prograde orbits around rapidly spinning SMBHs, while an optically selected sample will still be biased toward high $\left|\chi_{\bullet}\right|$, but much more weakly so, and may possess either a prograde or retrograde bias, depending on the specific optical emission mechanism. A consequence of this is that the quasi-thermal $\mathrm{X}$-ray luminosities in optically selected TDE distributions should be systematically lower than the corresponding X-ray luminosities in an X-ray-selected sample, since the former will have cooler disk temperatures, on average.

\section{Conclusions}

We have conducted Chandra X-ray observations of four optically selected TDEs long after the peak of their optical flares. In three cases, we detected late-time soft X-ray emission:
PTF09axc, PTF09ge, and ASASSN-14ae are best fit with unabsorbed $(0.3-7 \mathrm{keV})$ luminosities of $(3.2 \pm 0.2) \times 10^{42}$, $3.9_{-1.0}^{+1.1} \times 10^{41}$, and $9_{-5}^{+9} \times 10^{40} \mathrm{erg} \mathrm{s}^{-1}$, respectively. Our fourth target, PTF09djl, was undetected by Chandra, yielding an upper limit on its soft X-ray luminosity of $L_{\mathrm{X}}<3 \times$ $10^{41} \mathrm{erg} \mathrm{s}^{-1}$. Three of these observations represent the longest temporal baseline for X-ray observations of optically selected TDEs to date: PTF09axc and PTF09ge were observed roughly 8 yr after peak, while PTF09djl was observed roughly $10 \mathrm{yr}$ post-peak.

These TDEs exhibit a diversity of X-ray behavior at late times. The X-ray spectrum of PTF09ge is best fit by a very soft power law (and has similarities to the high-soft state of XRBs). In contrast, the X-ray spectrum of PTF09axc is best fit as a comparatively hard, nonthermal power law, quite unlike most TDEs seen at early times but not unlike several other TDEs seen at similarly late times, as shown in the literature, and more similar to the spectrum of an AGN or the low-hard state of an XRB. The TDE ASASSN-14ae does not have sufficient X-ray counts to determine the shape of its spectrum.

Our primary conclusions are as follows.

1. Late-time X-ray detections are further evidence that PTF09axc, PTF09ge, and, to a lesser extent, ASASSN14ae represent bona fide TDEs and not a peculiar type of nuclear SN explosion. The persistence of high X-ray luminosities $\approx 5-10 \mathrm{yr}$ post-peak also argues strongly against the presence of a thermal instability in TDE disks, as would be predicted by simple $\alpha$-disk theory.

2. We hypothesize that the marked spectral differences between PTF09axc and PTF09ge may have been caused by a late-time state change in PTF09axc to a low-hard state (in analogy to the state changes regularly observed in black hole XRBs). Radio follow-up observations of PTF09axc could test this hypothesis, as could continued X-ray monitoring of PTF09ge to investigate whether it also exhibits a state change to a (harder) power-law spectrum.

3. Assuming that our observations 4-9 yr after optical detection are not caused by short-lived flares, we conclude that most TDEs are persistently bright X-ray sources visible for at least a decade. This is in line with previous work. Overall, nine out of 10 TDEs observed in $\mathrm{X}$-ray at late times ( $\gtrsim 5 \mathrm{yr}$ after its discovery) were detected. This has implications for detection rates in nearfuture, wide-field X-ray surveys if deep enough earlytime X-ray observations of optically selected TDEs exist. For example, we find that the eROSITA instrument recently launched on the Spectrum Röntgen Gamma satellite could detect up to 1000 TDE flares per year if most low-mass SMBHs have near-maximal spins. However, the detection rate would be $170 \mathrm{yr}^{-1}$ if most SMBHs have zero spin and (in the Schwarzschild limit) further reduced to only $5 \mathrm{yr}^{-1}$ if $\mathrm{SMBHs}$ with masses below $10^{6} M_{\odot}$ are excluded.

4. We propose that there is often a delay between the peak optical and the X-ray emission in TDEs, such that optical and X-ray-selected TDEs are, in many cases, the same type of flare observed at different stages. For example, in X-ray-selected TDEs, the optical emission (e.g., from the circularization shock) may have already subsided below the level that can be detected above the nuclear region of the host galaxy. 
5. The persistence of a soft X-ray spectrum at late times (such as in PTF09ge) opens up the possibility of black hole spin determinations using continuum fitting techniques (S. Wen et al. 2020, in preparation). These were, in the past, primarily applied to black holes in soft-state XRBs (McClintock et al. 2014). Late-time X-ray observations will avoid, or at least minimize, theoretical uncertainties associated with early-time TDE disk modeling, such as generic disk tilts, significantly noncircular gas flows, and the presence of optically thick stellar debris on larger scales. The number of X-ray photons detected in the current observations of PTF09ge are, however, insufficient to attempt this exercise.

6. If the SMBHs responsible for TDEs possess appreciable spins, a flux-limited sample of TDEs will generally be biased toward an excess of prograde or retrograde disks. In an optically selected sample, the sign of this bias depends on the exact emission mechanism. Shock-powered optical emission (Piran et al. 2015) will exhibit a mild retrograde bias in the ELC regime and a mild prograde bias in the FLC regime. If instead, the optical emission is powered by reprocessed X-rays generated from a veiled inner accretion flow (Guillochon et al. 2014; Metzger \& Stone 2016), then prograde black hole spins are almost always favored, usually by a factor of a few. The X-ray-selected TDE samples have a very strong (1-2 orders of magnitude) bias for prograde orbits if most SMBHs are spinning rapidly.

We would like to thank the Chandra X-ray Observatory for approving and carrying out the observations presented in this paper and the anonymous referee for comments that helped improve the paper. P.G.J. acknowledges funding from the European Research Council under ERC Consolidator Grant agreement No. 647208 and discussions with Giacomo Cannizzaro. N.C.S. and A.G. acknowledge funding from Chandra GO 18700591. N.C.S. acknowledges additional funding from Chandra GO 20700515. B.D.M. acknowledges support from NASA through the Astrophysics Theory Program (grant No. NNX17AK43G). This research has made use of the NASA/ IPAC Extragalactic Database (NED), which is operated by the Jet Propulsion Laboratory, California Institute of Technology, under contract with the National Aeronautics and Space Administration.

Software: tool 1 XSPEC version 12.10.1 (HEASOFT), tool 2 Ciao version 4.10 developed by the Chandra X-ray Center (Fruscione et al. 2006).

\section{ORCID iDs}

P. G. Jonker (1) https://orcid.org/0000-0001-5679-0695

S. van Velzen (1) https://orcid.org/0000-0002-3859-8074

B. Metzger 누 https://orcid.org/0000-0002-4670-7509

\section{References}

Alexander, K. D., Wieringa, M. H., Berger, E., Saxton, R. D., \& Komossa, S. 2017, ApJ, 837, 153

Arcavi, I., Gal-Yam, A., Sullivan, M., et al. 2014, ApJ, 793, 38

Auchettl, K., Guillochon, J., \& Ramirez-Ruiz, E. 2017, ApJ, 838, 149

Bade, N., Komossa, S., \& Dahlem, M. 1996, A\&A, 309, L35

Bardeen, J. M., Press, W. H., \& Teukolsky, S. A. 1972, ApJ, 178, 347

Begelman, M. C., \& Pringle, J. E. 2007, MNRAS, 375, 1070

Berger, E., Zauderer, A., Pooley, G. G., et al. 2012, ApJ, 748, 36

Blagorodnova, N., Gezari, S., Hung, T., et al. 2017, ApJ, 844, 46

Bloom, J. S., Giannios, D., Metzger, B. D., et al. 2011, Sci, 333, 203
Bonnerot, C., Rossi, E. M., \& Lodato, G. 2017, MNRAS, 464, 2816

Bonnerot, C., Rossi, E. M., Lodato, G., \& Price, D. J. 2016, MNRAS, 455,2253

Bower, G. C., Metzger, B. D., Cenko, S. B., Silverman, J. M., \& Bloom, J. S. 2013, ApJ, 763, 84

Cannizzo, J. K., Lee, H. M., \& Goodman, J. 1990, ApJ, 351, 38

Cash, W. 1979, ApJ, 228, 939

Chan, C.-H., Piran, T., Krolik, J. H., \& Saban, D. 2019, ApJ, 881, 113

Chandra, P., Chevalier, R. A., Chugai, N., et al. 2012, ApJ, 755, 110

Chauhan, J., Miller-Jones, J. C. A., Anderson, G. E., et al. 2019, MNRAS, 488, L129

Chornock, R., Berger, E., Gezari, S., et al. 2014, ApJ, 780, 44

Coughlin, E. R., \& Begelman, M. C. 2014, ApJ, 781, 82

Czerny, B., \& Hryniewicz, K. 2011, A\&A, 525, L8

Dai, L., Escala, A., \& Coppi, P. 2013, ApJL, 775, L9

Dai, L., McKinney, J. C., \& Miller, M. C. 2015, ApJL, 812, L39

Dai, L., McKinney, J. C., Roth, N., Ramirez-Ruiz, E., \& Miller, M. C. 2018, ApJL, 859, L20

Donato, D., Cenko, S. B., Covino, S., et al. 2014, ApJ, 781, 59

Done, C., Davis, S. W., Jin, C., Blaes, O., \& Ward, M. 2012, MNRAS, 420, 1848

Dwarkadas, V. V., \& Gruszko, J. 2012, MNRAS, 419, 1515

Evans, I. N., Primini, F. A., Glotfelty, K. J., et al. 2010, ApJS, 189, 37

Falcke, H., Körding, E., \& Markoff, S. 2004, A\&A, 414, 895

Farrar, G. R., \& Gruzinov, A. 2009, ApJ, 693, 329

Farrar, G. R., \& Piran, T. 2014, arXiv:1411.0704

Fender, R., \& Belloni, T. 2004, ARA\&A, 42, 317

Fender, R. P., Belloni, T. M., \& Gallo, E. 2004, MNRAS, 355, 1105

Fragile, P. C. 2009, ApJL, 706, L246

Franchini, A., Lodato, G., \& Facchini, S. 2016, MNRAS, 455, 1946

Frank, J., \& Rees, M. J. 1976, MNRAS, 176, 633

Fruscione, A., McDowell, J. C., Allen, G. E., et al. 2006, Proc. SPIE, 6270 , $62701 \mathrm{~V}$

Garmire, G. P. 1997, BAAS, 29, 823

Generozov, A., Mimica, P., Metzger, B. D., et al. 2017, MNRAS, 464, 2481

Gezari, S., Cenko, S. B., \& Arcavi, I. 2017, ApJL, 851, L47

Gezari, S., Chornock, R., Rest, A., et al. 2012, Natur, 485, 217

Gezari, S., Heckman, T., Cenko, S. B., et al. 2009, ApJ, 698, 1367

Gezari, S., Martin, D. C., Milliard, B., et al. 2006, ApJL, 653, L25

Giannios, D., \& Metzger, B. D. 2011, MNRAS, 416, 2102

Gladstone, J. C., Roberts, T. P., \& Done, C. 2009, MNRAS, 397, 1836

Greiner, J., Schwarz, R., Zharikov, S., \& Orio, M. 2000, A\&A, 362, L25

Guillochon, J., Manukian, H., \& Ramirez-Ruiz, E. 2014, ApJ, 783, 23

Guillochon, J., \& Ramirez-Ruiz, E. 2013, ApJ, 767, 25

Guillochon, J., \& Ramirez-Ruiz, E. 2015, ApJ, 809, 166

Hasinger, G., \& van der Klis, M. 1989, A\&A, 225, 79

Hayasaki, K., Stone, N., \& Loeb, A. 2016, MNRAS, 461, 3760

Heckman, T. M., Ptak, A., Hornschemeier, A., \& Kauffmann, G. 2005, ApJ, 634, 161

Helene, O. 1984, NIMPA, 228, 120

Hills, J. G. 1975, Natur, 254, 295

Holoien, T. W.-S., Kochanek, C. S., Prieto, J. L., et al. 2016a, MNRAS, 455, 2918

Holoien, T. W.-S., Kochanek, C. S., Prieto, J. L., et al. 2016b, MNRAS, 463,3813

Holoien, T. W.-S., Prieto, J. L., Bersier, D., et al. 2014, MNRAS, 445, 3263

Homan, J., Wijnands, R., van der Klis, M., et al. 2001, ApJS, 132, 377

Hung, T., Gezari, S., Blagorodnova, N., et al. 2017, ApJ, 842, 29

Hung, T., Gezari, S., Cenko, S. B., et al. 2018, ApJS, 238, 15

Jiang, Y.-F., Blaes, O., Stone, J., \& Davis, S. W. 2019, ApJ, 885, 144

Jiang, Y.-F., Davis, S. W., \& Stone, J. M. 2016, ApJ, 827, 10

Jonker, P. G., Campana, S., Steeghs, D., et al. 2005, MNRAS, 361, 511

Jonker, P. G., Heida, M., Torres, M. A. P., et al. 2012, ApJ, 758, 28

Kalemci, E., Dinçer, T., Tomsick, J. A., et al. 2013, ApJ, 779, 95

Kaspi, S., Smith, P. S., Netzer, H., et al. 2000, ApJ, 533, 631

Kauffmann, G., \& Heckman, T. M. 2009, MNRAS, 397, 135

Kesden, M. 2012, PhRvD, 85, 024037

Khabibullin, I., Sazonov, S., \& Sunyaev, R. 2014, MNRAS, 437, 327

Kim, M., Kim, D.-W., Wilkes, B. J., et al. 2007, ApJS, 169, 401

Komossa, S., \& Bade, N. 1999, A\&A, 343, 775

Komossa, S., \& Greiner, J. 1999, A\&A, 349, L45

Komossa, S., Halpern, J., Schartel, N., et al. 2004, ApJL, 603, L17

Kraft, R. P., Burrows, D. N., \& Nousek, J. A. 1991, ApJ, 374, 344

Leloudas, G., Dai, L., Arcavi, I., et al. 2019, ApJ, 887, 218

Levan, A. J., Tanvir, N. R., Cenko, S. B., et al. 2011, Sci, 333, 199

Lin, D., Carrasco, E. R., Grupe, D., et al. 2011, ApJ, 738, 52 
Lin, D., Maksym, P. W., Irwin, J. A., et al. 2015, ApJ, 811, 43

Liu, F. K., Li, S., \& Chen, X. 2009, ApJL, 706, L133

Lodato, G., King, A. R., \& Pringle, J. E. 2009, MNRAS, 392, 332

Lodato, G., \& Rossi, E. M. 2011, MNRAS, 410, 359

Loeb, A., \& Ulmer, A. 1997, ApJ, 489, 573

Lu, W., \& Bonnerot, C. 2020, MNRAS, 492, 686

Maccarone, T. J. 2003, A\&A, 409, 697

Maccarone, T. J., \& Coppi, P. S. 2003, MNRAS, 338, 189

Maccarone, T. J., Gallo, E., \& Fender, R. 2003, MNRAS, 345, L19

Maksym, W. P., Lin, D., \& Irwin, J. A. 2014, ApJL, 792, L29

Mattila, S., Pérez-Torres, M., Efstathiou, A., et al. 2018, Sci, 361, 482

McClintock, J. E., Narayan, R., \& Steiner, J. F. 2014, SSRv, 183, 295

Merloni, A., \& Fabian, A. C. 2001, MNRAS, 321, 549

Merloni, A., Heinz, S., \& di Matteo, T. 2003, MNRAS, 345, 1057

Merritt, D., Alexander, T., Mikkola, S., \& Will, C. M. 2010, PhRvD, 81, 062002

Metzger, B. D., \& Stone, N. C. 2016, MNRAS, 461, 948

Miller, J. M., Kaastra, J. S., Miller, M. C., et al. 2015, Natur, 526, 542

Miller, M. C. 2015, ApJ, 805, 83

Nealon, R., Price, D. J., Bonnerot, C., \& Lodato, G. 2018, MNRAS, 474, 1737

Parfrey, K., Giannios, D., \& Beloborodov, A. M. 2015, MNRAS, 446, L61

Piran, T., Svirski, G., Krolik, J., Cheng, R. M., \& Shiokawa, H. 2015, ApJ, 806, 164

Predehl, P., \& Schmitt, J. H. M. M. 1995, A\&A, 293, 889

Rees, M. J. 1988, Natur, 333, 523

Roth, N., Kasen, D., Guillochon, J., \& Ramirez-Ruiz, E. 2016, ApJ, 827, 3

Rots, A. H., \& Budavári, T. 2011, ApJS, 192, 8

Saxton, C. J., Perets, H. B., \& Baskin, A. 2018, MNRAS, 474, 3307
Saxton, R. D., Read, A. M., Esquej, P., et al. 2012, A\&A, 541, A106

Saxton, R. D., Read, A. M., Komossa, S., et al. 2017, A\&A, 598, A29

Saxton, R. D., Read, A. M., Komossa, S., et al. 2019, A\&A, 630, A98

Sądowski, A. 2016, MNRAS, 459, 4397

Schlafly, E. F., \& Finkbeiner, D. P. 2011, ApJ, 737, 103

Shankar, F., Weinberg, D. H., \& Miralda-Escudé, J. 2009, ApJ, 690, 20

Shen, R.-F., \& Matzner, C. D. 2014, ApJ, 784, 87

Shiokawa, H., Krolik, J. H., Cheng, R. M., Piran, T., \& Noble, S. C. 2015, ApJ, 804, 85

Stone, N., \& Loeb, A. 2011, MNRAS, 412, 75

Stone, N., \& Loeb, A. 2012, PhRvL, 108, 061302

Stone, N., Sari, R., \& Loeb, A. 2013, MNRAS, 435, 1809

Stone, N. C., \& Metzger, B. D. 2016, MNRAS, 455, 859

Strubbe, L. E., \& Quataert, E. 2009, MNRAS, 400, 2070

Tchekhovskoy, A., Metzger, B. D., Giannios, D., \& Kelley, L. Z. 2014, MNRAS, 437, 2744

Ulmer, A. 1999, ApJ, 514, 180

van Velzen, S., Farrar, G. R., Gezari, S., et al. 2011a, ApJ, 741, 73

van Velzen, S., Frail, D. A., Körding, E., \& Falcke, H. 2013, A\&A, 552, A5

van Velzen, S., Gezari, S., Cenko, S. B., et al. 2019a, ApJ, 872, 198

van Velzen, S., Körding, E., \& Falcke, H. 2011b, MNRAS, 417, L51

van Velzen, S., Stone, N. C., Metzger, B. D., et al. 2019b, ApJ, 878, 82

Vaughan, S., Edelson, R., \& Warwick, R. S. 2004, MNRAS, 349, L1

Wevers, T., Pasham, D. R., van Velzen, S., et al. 2019a, MNRAS, 488, 4816

Wevers, T., Stone, N. C., van Velzen, S., et al. 2019b, MNRAS, 487, 4136

Wevers, T., van Velzen, S., Jonker, P. G., et al. 2017, MNRAS, 471, 1694

Woo, J.-H., \& Urry, C. M. 2002, ApJ, 579, 530

Yuan, W., Zhang, C., Feng, H., et al. 2015, arXiv:1506.07735 